\title{
Self-harm with suicidal and non-suicidal intent in young people in sub-Saharan Africa: a systematic review
}

Emmanuel N-B Quarshie ${ }^{1 *}$, Mitch G. Waterman ${ }^{2}$ and Allan O. House ${ }^{3}$

\begin{abstract}
Background: Self-harm, whether attributed to suicidal or non-suicidal motives, is associated with several poor outcomes in young people, including eventual suicide. Much of our understanding of self-harm in young people is based on literature from Europe (particularly, the UK), North America, and Australia. We aimed to synthesise the available evidence on prevalence, the commonly reported self-harm methods, correlates, risk and protective factors, and reasons for self-harm, in adolescents (aged 10-25 years) in sub-Saharan Africa.
\end{abstract}

Method: We searched MEDLINE, PsycINFO, PubMed, African Journals OnLine, and African Index Medicus for records from 1950 through August 2019, without language restrictions. We supplemented the database searches by searching relevant portals for postgraduate theses, reference harvesting, contacting authors for unpublished studies, and hand searching relevant print sources. We applied narrative synthesis to the evidence.

Results: Seventy-four studies from 18 sub-Saharan African countries met the inclusion criteria. The median lifetime prevalence estimate was 10.3\% (interquartile range [IQR] 4.6\% - 16.1\%); median 12-month prevalence estimate was 16.9\% (IQR: 11.5\% - 25.5\%); median 6-month prevalence estimate was 18.2\% (IQR: 12.7\% - 21.8\%); and the median 1-month prevalence estimate was 3.2\% (IQR: 2.5-14.8\%). Studies from Western sub-Saharan Africa reported the highest 12-month prevalence estimates (median $=24.3 \%$; IQR $=16.9 \%-27.9 \%$ ). Clinical samples commonly reported overdose, whereas self-cutting was most commonly reported in non-clinical samples. Academic failure, sexual, emotional, and physical abuse, romantic relationship problems, family conflict, depression, and previous self-harm were identified as key correlates of self-harm. No study reported protective factors against self-harm.

Conclusion: Variation in estimates was explained by small sample sizes and variation in definitions and measures used. Exploration of associations, risks and protective factors was based upon concepts and measures derived from high income countries. More detailed and culturally sensitive research is needed to understand the context-specific risks and protective factors for self-harm in adolescents in sub-Saharan Africa.

Keywords: Adolescents, Attempted suicide, Self-harm, Sub-Saharan Africa, Suicide

\footnotetext{
* Correspondence: enquarshie@ug.edu.gh; enquarshie@gmail.com

'Department of Psychology, University of Ghana, P.O. Box LG 84, Legon, Accra, Ghana

Full list of author information is available at the end of the article
}

(C) The Author(s). 2020 Open Access This article is licensed under a Creative Commons Attribution 4.0 International License, which permits use, sharing, adaptation, distribution and reproduction in any medium or format, as long as you give appropriate credit to the original author(s) and the source, provide a link to the Creative Commons licence, and indicate if changes were made. The images or other third party material in this article are included in the article's Creative Commons licence, unless indicated otherwise in a credit line to the material. If material is not included in the article's Creative Commons licence and your intended use is not permitted by statutory regulation or exceeds the permitted use, you will need to obtain permission directly from the copyright holder. To view a copy of this licence, visit http://creativecommons.org/licenses/by/4.0/ The Creative Commons Public Domain Dedication waiver (http://creativecommons.org/publicdomain/zero/1.0/) applies to the data made available in this article, unless otherwise stated in a credit line to the data. 


\section{Background}

The World Health Organisation (WHO) defines selfharm as "an act with non-fatal outcome in which an individual deliberately initiates a non-habitual behaviour, that without intervention from others will cause selfharm, or deliberately ingests a substance in excess of the prescribed or generally recognised therapeutic dosage, and which is aimed at realising changes that the person desires via the actual or expected physical consequences" $[1,2]$.

This definition does not distinguish acts of self-harm according to intent, and for brevity in this review we use the term "self-harm" to refer to acts that are attributed to suicidal and non-suicidal motivations. Self-harm among young people is a recognised problem in the mental health of populations in high income countries, where it is associated with a number of poor outcomes including eventual suicide [3, 4]. By comparison we know little about self-harm in young people in subSaharan Africa; instead much of our understanding is based on extrapolation from literature from Europe, particularly the UK, North America, and Australia [5-8]. Earlier regional reviews have either included only a few selected studies of young people from Africa, [9-11] or included only studies involving adult population samples from the region [12]. Thus, we have found no existing review that has systematically appraised the available published and unpublished evidence specifically on selfharm among adolescents in countries in sub-Saharan Africa.

The aims of the present review were to:

1) Describe the lifetime, 12-month, 6-month, and 1month prevalence estimates of self-harm in young people (aged 10-25 years) across sub-Saharan Africa.

2) Describe the commonest methods of self-harm in young people identified across the previous studies.

3) Identify the commonest associations, risks, and protective factors associated with self-harm in young people observed in previous studies across sub-Saharan Africa.

4) Describe the self-reported reasons for self-harm in young people across sub-Saharan Africa.

\section{Methods}

This systematic review followed PRISMA guidelines [13] (see Additional files 1 and 2). We searched MEDLINE, PsycINFO, PubMed, African Journals OnLine, African Index Medicus, and the South African national Electronic Theses and Dissertations (SA-ETD) portal, (Additional file 3) between January 1950 and August 2019, without language restrictions. The geographic search filter included names of the countries in English and languages relevant to the countries [14]. When a country's name had changed after 1950 [15], both current and earlier names were included. We searched grey literature, institutional and organisational reports and national and international government reports (e.g., WHO, World Bank, UNICEF, UNDP) [16]. We hand searched edited books, the West African Journal of Medicine, Ghana Medical Journal, South African Medical Journal, Ethiopian Medical Journal, and the East African Medical Journal. We contacted academics with a research interest in self-harm and authors who published two or more articles on self-harm identified during our review (see Additional file 4). Reference harvesting used Science Citation Index, Google Scholar, and manual search. Criteria for inclusion and exclusion of studies are shown in Table 1.

We used EndNote (version X9.2) to collate and handle the identified records. All records were screened for eligibility by reading the titles, abstracts, methods, and results sections by EQ, with consensus discussion of 10 $20 \%$ of studies with co-authors. Appraisal of the methodological quality of records used the mixed method appraisal tool (MMAT) [17]. There was substantial heterogeneity across the studies. We applied narrative synthesis to the evidence in the final set of studies; we present the prevalence estimates as median values and interquartile ranges (IQRs). We report pooled estimates (median and IQR) to aid presentation of the data, but note should be taken of the substantial heterogeneity in studies included $[18,19]$.

\section{Results}

Seventy-four studies involving adolescents aged 10-25 years were included after removing duplicates - one national report on adolescent health behaviour, seven (9.4\%) postgraduate theses, one book chapter and 65 (87.8\%) peer-reviewed articles published in indexed academic journals (see Additional file 5).

\section{Characteristics of included studies}

The majority of the studies, 54 (73\%), used the terms "suicide attempt", "suicidal attempt", or "attempted suicide", to describe self-harm. Although included studies distinguished between suicidal self-harm and nonsuicidal self-harm in their findings none of the studies indicated if a definition or explanation of the core question was provided to participants [20]. Because we wanted to include all acts that meet the WHO definition, which does not include a requirement for a specific intent, given the contention about the soundness of the distinction between self-reported suicidal and nonsuicidal acts [21], which is likely to be a particular problem in countries where suicide is illegal and where different languages may not readily reflect the distinction, and as individual suicide risk is known to reside in all 
Table 1 Summary of inclusion and exclusion criteria

\begin{tabular}{ll}
\hline Criterion & Include \\
\hline $\begin{array}{l}\text { Definition and } \\
\text { measurement of } \\
\text { self-harm }\end{array}$ & $\begin{array}{l}\text { - Studies with clear definitions of self-harm (or alternative term or } \\
\text { concept used) as an intentional act of self-inflicted injury or poi- } \\
\text { soning, in addition to clear means of case identification, assess- } \\
\text { ment or measurement. }\end{array}$ \\
& \\
Prevalence & $\begin{array}{l}\text { - Studies with specified time frames within which prevalence of } \\
\text { estimate }\end{array}$ \\
& self-harm was assessed.
\end{tabular}

Exclude

- Studies focused on unintended self-harm behaviours (e.g., smoking, drink-driving, eating disorders etc.). - Studies focused on intended self-harm with socially sanctioned motives (e.g., scarification, manhood rituals, 'body enhancement', religious fasting, hunger strikes etc.).

- Studies focused on intended self-harm behaviours not approved by the broader sociocultural context but are sanctioned by the subcultures (e.g., cult groups, Goth subcultures, Emo subcultures etc.) within which they occur.

- Studies focused on suicidal ideations, self-harm thoughts, or threats, as these do not necessarily translate into or represent acts of self-harm.

- Studies focused on suicide (self-inflicted death).

- If prevalence estimates cannot be determined within a clear time frame;

- If there is no clear indication of sample size and population denominator.

- Studies focused on adolescents in prisons or borstal institutions, unless control groups in such studies allow for the evaluation of risk and protective factors of self-harm in adolescents. estimate

Setting

Study Designs
- Studies with primary focus on self-harm conducted within nonclinical contexts (i.e., general population, community, schoolbased, households / neighbourhoods, street-connected settings etc.) in countries within sub-Saharan Africa.

- Studies conducted in clinical contexts focused on self-harm as the main presenting condition.

- Clinical studies concerned with self-harm as the primary condition (but not as comorbid condition, e.g., self-harm in HIV/AIDS or epilepsy).

Participants $\quad$ - Studies reporting prevalence estimates of self-harm involving participants aged between 10 and 25 years.

- Studies reporting on the associates, risk and protective factors related to self-harm, methods of self-harm used, and reported reasons for self-harm involving participants aged 10 and 25 years with a personal self-harm history at the time of assessment for the study.

- Studies with wide age range but majority (90\% or more) of the participants are within the age bracket of 10-25 years.

\footnotetext{
- Studies with focus on self-harm which address at least one of the four specified objectives of this review using: (1) quantitative methods (i.e., school-based, household-based, population/community-based cross-sectional survey; census; retrospective or prospective descriptive cohort designs; case controls; case reports; randomised controlled trials, and analytic cohort designs); or (2) qualitative methods (e.g., interviews, focus groups etc.); or (3) retrospective reviews of clinical records.

- Cross-national studies involving countries in sub-Saharan Africa and other countries outside the sub-region, which stratify and link the results to the included countries. In such instances, the specified results related to the sub-Saharan African countries were included in this review.
}

- Adolescents with pervasive developmental disorders, cancer, insulin-dependent diabetes, epilepsy or HIV/AIDS adolescent patients, unless control groups in such studies allow for the evaluation of risk and protective factors of self-harm. - Studies involving participants within wide age ranges with the study results not disaggregated by age, making it impossible to link specific results to participants age 10-25 years, and where participants are stratified by age but with participants aged $10-25$ years constituting less than $90 \%$ of the total sample which did not specifically link the reported prevalence estimates, identified risks or associates of selfharm, protective factors, methods of self-harm, or the identified reasons for self-harm to young people aged 10-25 years.

- Studies based on the same dataset reported in an earlier publication included in this review.

- Systematic reviews, commentaries, editorials, opinion pieces, correspondence, and articles not based on data.

- Where full text of the identified article was unavailable or could not be accessed.

- Cross-national studies involving countries in sub-Saharan Africa and other countries outside the sub-region, which did not stratify or link the results to the respective included countries. acts of self-harm regardless of attribution, we have included all studies in our estimates of prevalence where it was clear that self-harm was the question put to participants, regardless of apparent intent.

Data were available from all four geographical subregions of sub-Saharan Africa. The majority (44.6\%) were from five countries within Southern sub-Saharan Africa (eSwatini, Mozambique, Namibia, South Africa, and Zambia) - South Africa ranked the highest with more than half $(n=37)$ of the total included records; $30.4 \%$ were based on data from six Western sub-Saharan African countries (Benin, Ghana, Ivory Coast, Mauritania, Nigeria, and Togo); $1.1 \%$ from CongoBrazzaville in Central sub-Saharan Africa; and 23.9\% of data obtained from six countries within Eastern subSaharan Africa (Ethiopia, Malawi, Rwanda, Seychelles, 
Table 2 Prevalence Estimates of Self-harm (by year and country of publication)

\begin{tabular}{|c|c|c|c|c|c|c|c|}
\hline \multirow{2}{*}{$\begin{array}{l}\text { Author } \\
\text { (year) } \\
\text { Country }\end{array}$} & \multirow[t]{2}{*}{ Term } & \multirow[t]{2}{*}{$\begin{array}{l}\text { Setting } \\
\text { (sample) }\end{array}$} & \multicolumn{4}{|l|}{$\begin{array}{l}\text { Prevalence } \\
\text { estimate }\end{array}$} & \multirow[t]{2}{*}{$\begin{array}{l}\text { Study } \\
\text { quality }\end{array}$} \\
\hline & & & Lifetime & 12-month & 6-month & 1-month & \\
\hline $\begin{array}{l}\text { Flisher et al. } \\
\text { (1993) [34]. } \\
\text { South Africa. }\end{array}$ & Attempted suicide & $\begin{array}{l}\text { School } \\
\text { (7340) }\end{array}$ & - & $\begin{array}{l}\mathrm{OV}=572 / 7340 \\
(7.8 \%)\end{array}$ & - & - & $2 / 5$ \\
\hline $\begin{array}{l}\text { Kebede \& Ketsela } \\
\text { (1993) [35]. } \\
\text { Ethiopia. }\end{array}$ & Attempted suicide & $\begin{array}{l}\text { School } \\
(519)\end{array}$ & $\begin{array}{l}\text { OV }=74 / 519 \\
(14.3 \%) \\
F=32 / 232 \\
(13.8 \%) \\
M=42 / 287 \\
(14.6 \%)\end{array}$ & - & - & - & $5 / 5$ \\
\hline $\begin{array}{l}\text { Peltzer et al. } \\
\text { (2000) [36]. } \\
\text { South Africa. }\end{array}$ & Attempted suicide & $\begin{array}{l}\text { School } \\
\text { (366) }\end{array}$ & $\begin{array}{l}\mathrm{OV}=46 / 366 \\
(12.6 \%)\end{array}$ & - & - & - & $3 / 5$ \\
\hline $\begin{array}{l}\text { Madu \& Matla } \\
\text { (2003) [37]. } \\
\text { South Africa. }\end{array}$ & Attempted suicide & $\begin{array}{l}\text { School } \\
\text { (435) }\end{array}$ & $\begin{array}{l}\mathrm{OV}=91 / 435 \\
(21 \%) \\
F=43 / 243(18 \%) \\
M=48 / 192 \\
(25 \%)\end{array}$ & - & - & - & $4 / 5$ \\
\hline $\begin{array}{l}\text { Wild et al. } \\
\text { (2004) [38]. } \\
\text { South Africa. }\end{array}$ & Suicidal attempt & $\begin{array}{l}\text { School } \\
\text { (939) }\end{array}$ & - & $\begin{array}{l}\text { OV }=95 / 939 \\
(10 \%) \\
F=69 / 519(13.3 \%) \\
M=26 / 420(6.2 \%)\end{array}$ & - & - & $4 / 5$ \\
\hline $\begin{array}{l}\text { Sommer } \\
\text { (2005) [39]. } \\
\text { Cross-national } \\
\text { (South Africa } \\
\text { \& Germany) }\end{array}$ & Suicidal behaviour & $\begin{array}{l}\text { School } \\
\text { (299) }\end{array}$ & $\begin{array}{l}O V=48 / 299 \\
(16.1 \%) \\
F=38 / 185 \\
(20.5 \%) \\
M=10 / 114 \\
(8.8 \%)\end{array}$ & - & - & - & $2 / 5$ \\
\hline $\begin{array}{l}\text { Flisher et al. } \\
\text { (2006) [40]. } \\
\text { South Africa. }\end{array}$ & Suicidal attempt & $\begin{array}{l}\text { School } \\
(10669)\end{array}$ & - & $\begin{array}{l}\text { OV }=9.1 \% \\
F=691 / 6066 \\
(11.4 \%) \\
M=313 / 4603 \\
(6.8 \%)\end{array}$ & - & - & $3 / 5$ \\
\hline $\begin{array}{l}\text { Shiferaw et al. } \\
\text { (2006) [41]. } \\
\text { Ethiopia. }\end{array}$ & Suicidal attempt & $\begin{array}{l}\text { School } \\
(667)\end{array}$ & $\begin{array}{l}\mathrm{OV}=44 / 667 \\
(6.6 \%)\end{array}$ & $\begin{array}{l}\text { OV }=39 / 667 \\
(5.8 \%) \\
F=11 / 155(7.1 \%) \\
M=28 / 512(5.5 \%)\end{array}$ & - & - & $2 / 5$ \\
\hline $\begin{array}{l}\text { Omigbodun et al. } \\
\text { (2008) [20]. } \\
\text { Nigeria. }\end{array}$ & Attempted suicide & $\begin{array}{l}\text { School } \\
(1429)\end{array}$ & - & $\begin{array}{l}\text { OV }=167 / 1429 \\
(11.7 \%) \\
F=87 / 702(12.4 \%) \\
M=80 / 727(11 \%)\end{array}$ & - & - & $4 / 5$ \\
\hline $\begin{array}{l}\text { Peltzer } \\
\text { (2008) [42]. } \\
\text { South Africa }\end{array}$ & Suicide attempt & $\begin{array}{l}\text { School } \\
(1157)\end{array}$ & - & $\begin{array}{l}\text { OV }=278 / 1157 \\
(24 \%)\end{array}$ & - & - & $4 / 5$ \\
\hline $\begin{array}{l}\text { Mashego \& Madu (2009) } \\
\text { [43]. } \\
\text { South Africa. }\end{array}$ & Suicidal behaviour & $\begin{array}{l}\text { School } \\
(142)\end{array}$ & $\begin{array}{l}\mathrm{OV}=21 / 142 \\
(14.8 \%) \\
F=14 / 86(16.3 \%) \\
M=7 / 56(12.5 \%)\end{array}$ & - & - & - & $4 / 5$ \\
\hline $\begin{array}{l}\text { Kinyanda et al. } \\
\text { (2011) [28]. } \\
\text { Uganda. }\end{array}$ & $\begin{array}{l}\text { Self-injury } \\
\& \\
\text { Suicide attempt }\end{array}$ & $\begin{array}{l}\text { Community } \\
\text { (897) }\end{array}$ & $\begin{array}{l}\text { Self-injury: } \\
\text { 13/897 (1.4\%) } \\
\text { Suicide attempt: } \\
15 / 897(1.7 \%)\end{array}$ & - & - & - & $5 / 5$ \\
\hline $\begin{array}{l}\text { Nanewortor } \\
\text { (2011) [44]. } \\
\text { Ghana }\end{array}$ & Attempted suicide & $\begin{array}{l}\text { School } \\
\text { (383) }\end{array}$ & $\begin{array}{l}\mathrm{OV}=31 / 383 \\
(8.1 \%)\end{array}$ & - & - & - & $4 / 5$ \\
\hline $\begin{array}{l}\text { Campbell } \\
\text { (2012) [45]. } \\
\text { South Africa }\end{array}$ & Attempted suicide & $\begin{array}{l}\text { School } \\
(1033)\end{array}$ & $\begin{array}{l}\text { OV }=129 / 1033 \\
(12.5 \%) \\
F=100 / 552 \\
(18.1 \%) \\
M=26 / 437\end{array}$ & - & - & - & $3 / 5$ \\
\hline
\end{tabular}


Table 2 Prevalence Estimates of Self-harm (by year and country of publication) (Continued)

\begin{tabular}{|c|c|c|c|c|c|c|c|}
\hline \multirow{2}{*}{$\begin{array}{l}\text { Author } \\
\text { (year) } \\
\text { Country }\end{array}$} & \multirow[t]{2}{*}{ Term } & \multirow[t]{2}{*}{$\begin{array}{l}\text { Setting } \\
\text { (sample) }\end{array}$} & \multicolumn{4}{|l|}{$\begin{array}{l}\text { Prevalence } \\
\text { estimate }\end{array}$} & \multirow[t]{2}{*}{$\begin{array}{l}\text { Study } \\
\text { quality }\end{array}$} \\
\hline & & & Lifetime & 12-month & 6-month & 1-month & \\
\hline & & & $(5.9 \%)$ & & & & \\
\hline $\begin{array}{l}\text { Swahn et al. } \\
\text { (2012) [30]. } \\
\text { Uganda. }\end{array}$ & Suicidal attempt & $\begin{array}{l}\text { Community } \\
\text { (457) }\end{array}$ & - & $\begin{array}{l}\text { OV }=90 / 457 \\
(19.8 \%) \\
F=67 / 313(21.4 \%) \\
M=23 / 144 \\
(16.2 \%)\end{array}$ & - & - & $4 / 5$ \\
\hline $\begin{array}{l}\text { van Niekerk et al. } \\
\text { (2012) [46]. } \\
\text { South Africa. }\end{array}$ & Suicidal attempt & $\begin{array}{l}\text { University } \\
\text { (810) }\end{array}$ & $\begin{array}{l}\mathrm{OV}=47 / 810 \\
(5.8 \%)\end{array}$ & - & - & - & $3 / 5$ \\
\hline $\begin{array}{l}\text { Vawda } \\
\text { (2012) [47]. } \\
\text { South Africa }\end{array}$ & Suicide attempt & $\begin{array}{l}\text { School } \\
(219)\end{array}$ & $\begin{array}{l}\text { OV }=12 / 219 \\
(5.5 \%)\end{array}$ & - & - & - & $3 / 5$ \\
\hline $\begin{array}{l}\text { Gage } \\
\text { (2013) [25]. } \\
\text { Ethiopia }\end{array}$ & Suicide attempt & $\begin{array}{l}\text { Community } \\
\text { (2709) }\end{array}$ & - & - & $\begin{array}{l}\text { OV }=62 / 2709 \\
(2.3 \%)\end{array}$ & - & $4 / 5$ \\
\hline $\begin{array}{l}\text { Muula et al. } \\
\text { (2013) [48]. } \\
\text { Zambia. }\end{array}$ & $\begin{array}{l}\text { Self-inflicted serious } \\
\text { injury }\end{array}$ & $\begin{array}{l}\text { School } \\
(2136)\end{array}$ & - & $\begin{array}{l}\mathrm{OV}=254 / 2136 \\
(11.9 \%)\end{array}$ & - & - & $5 / 5$ \\
\hline $\begin{array}{l}\text { Shilubane et al. } \\
\text { (2013) [49]. } \\
\text { South Africa. }\end{array}$ & Suicidal attempt & $\begin{array}{l}\text { School: } \\
\text { 2002 SAYRBS } \\
(10549) \\
2008 \text { SAYRBS } \\
(10097)\end{array}$ & - & - & $\begin{array}{l}2002 \text { SAYRBS: } \\
\text { OV }=18.5 \% \\
F=19.5 \% \\
M=17.3 \% \\
2008 \text { SAYRBS: } \\
\text { OV }=21.8 \% \\
F=22.7 \% \\
M=20.8 \%\end{array}$ & - & $5 / 5$ \\
\hline $\begin{array}{l}\text { van Rooyen } \\
\text { (2013) [50]. } \\
\text { South Africa }\end{array}$ & Deliberate self-harm & $\begin{array}{l}\text { University } \\
\text { (603) }\end{array}$ & $\begin{array}{l}\mathrm{OV}=291 / 603 \\
(48.3 \%)\end{array}$ & $\begin{array}{l}\mathrm{OV}=223 / 6033 \\
(7.0 \%)\end{array}$ & - & - & $3 / 5$ \\
\hline $\begin{array}{l}\text { Cheng et al. } \\
\text { (2014) [33]. } \\
\text { Cross-national (Nigeria, } \\
\text { South Africa, China, } \\
\text { India, USA). }\end{array}$ & Suicidal attempt & $\begin{array}{l}\text { Community: } \\
\text { Nigeria } \\
\text { (449) } \\
\text { South Africa } \\
\text { (496) }\end{array}$ & - & $\begin{array}{l}\text { Nigeria: } \\
\text { OV=73/449 } \\
(16.3 \%) \\
F=33 / 229(14.3 \%) \\
M=40 / 220 \\
(18.3 \%) \\
\text { South Africa: } \\
O V=54 / 496 \\
(10.9 \%) \\
F=22 / 224(10 \%) \\
M=32 / 272 \\
(11.8 \%)\end{array}$ & - & - & $4 / 5$ \\
\hline $\begin{array}{l}\text { Chinawa et al. } \\
\text { (2014) [51]. } \\
\text { Nigeria. }\end{array}$ & Attempted suicide & $\begin{array}{l}\text { School } \\
\text { (764) }\end{array}$ & - & $\begin{array}{l}\mathrm{OV}=96 / 764 \\
(12.5 \%)\end{array}$ & - & - & $2 / 5$ \\
\hline $\begin{array}{l}\text { Lippi } \\
\text { (2014) [52]. } \\
\text { South Africa. }\end{array}$ & Deliberate self-harm & $\begin{array}{l}\text { University } \\
(603)\end{array}$ & $\begin{array}{l}\text { OV }=278 / 603 \\
(46.1 \%) \\
F=219 / 483 \\
(45.3 \%) \\
M=59 / 120 \\
(49.2 \%)\end{array}$ & $\begin{array}{l}\mathrm{OV}=216 / 603 \\
(35.8 \%)\end{array}$ & - & - & $3 / 5$ \\
\hline $\begin{array}{l}\text { Penning \& Collings } \\
\text { (2014) [53]. } \\
\text { South Africa. }\end{array}$ & Self-injury & $\begin{array}{l}\text { School } \\
\text { (716) }\end{array}$ & - & $\begin{array}{l}\text { OV }=20 / 716 \\
(2.8 \%)\end{array}$ & - & - & $5 / 5$ \\
\hline $\begin{array}{l}\text { Randall et al. } \\
\text { (2014) [54]. } \\
\text { Benin. }\end{array}$ & Attempted suicide & $\begin{array}{l}\text { School } \\
(2690)\end{array}$ & - & $\begin{array}{l}\mathrm{OV}=761 / 2690 \\
(28.3 \%)\end{array}$ & - & - & $5 / 5$ \\
\hline $\begin{array}{l}\text { Shilubane et al. } \\
\text { (2014) [55]. } \\
\text { South Africa. }\end{array}$ & Suicide attempt & $\begin{array}{l}\text { School } \\
\text { (591) }\end{array}$ & - & - & $\begin{array}{l}\mathrm{OV}=134 / 591 \\
(22.7 \%) \\
F=52 / 297\end{array}$ & - & $4 / 5$ \\
\hline
\end{tabular}


Table 2 Prevalence Estimates of Self-harm (by year and country of publication) (Continued)

\begin{tabular}{|c|c|c|c|c|c|c|c|}
\hline \multirow{2}{*}{$\begin{array}{l}\text { Author } \\
\text { (year) } \\
\text { Country }\end{array}$} & \multirow[t]{2}{*}{ Term } & \multirow[t]{2}{*}{$\begin{array}{l}\text { Setting } \\
\text { (sample) }\end{array}$} & \multicolumn{4}{|l|}{$\begin{array}{l}\text { Prevalence } \\
\text { estimate }\end{array}$} & \multirow[t]{2}{*}{$\begin{array}{l}\text { Study } \\
\text { quality }\end{array}$} \\
\hline & & & Lifetime & 12-month & 6-month & 1-month & \\
\hline & & & & & $\begin{array}{l}(18.2 \%) \\
M=77 / 294 \\
(27 \%)\end{array}$ & & \\
\hline $\begin{array}{l}\text { Cluver et al. } \\
\text { (2015) [56]. } \\
\text { South Africa }\end{array}$ & Suicide attempt & $\begin{array}{l}\text { Community } \\
(3401)\end{array}$ & - & - & - & $\begin{array}{l}\text { OV }=111 / 3401 \\
(3.3 \%) \\
F=79 / 1926 \\
(4.4 \%) \\
M=32 / 1475 \\
(2.2 \%)\end{array}$ & $4 / 5$ \\
\hline $\begin{array}{l}\mathrm{Ng} \text { et al. } \\
\text { (2015) [57]. } \\
\text { Rwanda }\end{array}$ & Suicidal behaviour & $\begin{array}{l}\text { Community } \\
(237)\end{array}$ & - & - & $\begin{array}{l}\mathrm{OV}=30 / 237 \\
(12.6 \%)\end{array}$ & - & $5 / 5$ \\
\hline $\begin{array}{l}\text { Giru } \\
\text { (2016) [58]. } \\
\text { Ethiopia }\end{array}$ & Suicide attempt & $\begin{array}{l}\text { School } \\
(722)\end{array}$ & $\begin{array}{l}\text { OV }=90 / 722 \\
(12.5 \%) \\
F=47 / 336(14 \%) \\
M=43 / 386 \\
(11.1 \%)\end{array}$ & - & - & $\begin{array}{l}O V=14 / 722 \\
(1.9 \%) \\
F=10 / 336(3 \%) \\
M=4 / 386(1 \%)\end{array}$ & $3 / 5$ \\
\hline $\begin{array}{l}\text { Shaikh et al. } \\
\text { (2016) [59]. } \\
\text { Malawi. }\end{array}$ & Suicide attempt & $\begin{array}{l}\text { School } \\
(2225)\end{array}$ & - & $\begin{array}{l}\text { OV }=287 / 2225 \\
(12.9 \%) \\
F=157 / 1188 \\
(13.2 \%) \\
M=130 / 1037 \\
(12.4 \%)\end{array}$ & - & - & $5 / 5$ \\
\hline $\begin{array}{l}\text { van der Walt } \\
\text { (2016) [60]. } \\
\text { South Africa }\end{array}$ & Self-harm & $\begin{array}{l}\text { University } \\
\text { (201) }\end{array}$ & $\begin{array}{l}\text { OV = 39/201 } \\
(19.4 \%)\end{array}$ & - & - & - & $3 / 5$ \\
\hline $\begin{array}{l}\text { Akanni et al. } \\
\text { (2017) [61]. } \\
\text { Nigeria }\end{array}$ & Attempted suicide & $\begin{array}{l}\text { School } \\
(300)\end{array}$ & - & $\begin{array}{l}\text { OV }=17 / 300 \\
(5.7 \%) \\
F=8 / 135(5.9 \%) \\
M=9 / 165(5.5 \%)\end{array}$ & - & - & $2 / 5$ \\
\hline $\begin{array}{l}\text { Asante et al. } \\
\text { (2017) [62]. } \\
\text { Ghana }\end{array}$ & Suicide attempt & $\begin{array}{l}\text { School } \\
\text { (1973) }\end{array}$ & - & $\begin{array}{l}\text { OV }=438 / 1973 \\
(22.2 \%) \\
F=213 / 908 \\
(23.5 \%) \\
M=225 / 1065 \\
(21.1 \%)\end{array}$ & - & - & $5 / 5$ \\
\hline $\begin{array}{l}\text { Asante \& Meyer-Weitz } \\
\text { (2017) [31]. } \\
\text { Ghana. }\end{array}$ & Suicidal attempt & $\begin{array}{l}\text { Community } \\
\text { (227) }\end{array}$ & - & - & - & $\begin{array}{l}\text { OV }=60 / 227 \\
(26.4 \%) \\
F=36 / 105 \\
(37.5 \%) \\
M=24 / 122 \\
(20.3 \%)\end{array}$ & $4 / 5$ \\
\hline $\begin{array}{l}\text { James et al. } \\
\text { (2017) [63]. } \\
\text { South Africa. }\end{array}$ & Suicidal attempt & $\begin{array}{l}\text { School } \\
(10997)\end{array}$ & - & - & $\mathrm{OV}=17.8 \%$ & - & $4 / 5$ \\
\hline $\begin{array}{l}\text { Nyandindi } \\
\text { (2017) [64]. } \\
\text { Tanzania }\end{array}$ & Suicide attempt & $\begin{array}{l}\text { School } \\
(3793)\end{array}$ & - & $\begin{array}{l}\text { OV }=436 / 3793 \\
(11.5 \%) \\
F=230 / 1931 \\
(11.9 \%) \\
M=192 / 1862 \\
(10.3 \%)\end{array}$ & - & - & $5 / 5$ \\
\hline $\begin{array}{l}\text { Peltzer \& Pengpid } \\
\text { (2017) [65]. } \\
\text { Namibia }\end{array}$ & Suicide attempt & $\begin{array}{l}\text { School } \\
(4531)\end{array}$ & - & $\begin{array}{l}\text { OV }=1029 / 4531 \\
(22.7 \%) \\
F=604 / 2406 \\
(27.4 \%) \\
M=577 / 2125 \\
(24.5 \%)\end{array}$ & - & - & $4 / 5$ \\
\hline $\begin{array}{l}\text { Stansfeld et al. } \\
\text { (2017) [66]. }\end{array}$ & Suicide attempt & $\begin{array}{l}\text { School } \\
(1034)\end{array}$ & - & $\begin{array}{l}\mathrm{OV}=139 / 1034 \\
(13.4 \%)\end{array}$ & - & - & $4 / 5$ \\
\hline
\end{tabular}


Table 2 Prevalence Estimates of Self-harm (by year and country of publication) (Continued)

\begin{tabular}{|c|c|c|c|c|c|c|c|}
\hline \multirow{2}{*}{$\begin{array}{l}\text { Author } \\
\text { (year) } \\
\text { Country }\end{array}$} & \multirow[t]{2}{*}{ Term } & \multirow[t]{2}{*}{$\begin{array}{l}\text { Setting } \\
\text { (sample) }\end{array}$} & \multicolumn{4}{|l|}{$\begin{array}{l}\text { Prevalence } \\
\text { estimate }\end{array}$} & \multirow[t]{2}{*}{$\begin{array}{l}\text { Study } \\
\text { quality }\end{array}$} \\
\hline & & & Lifetime & 12-month & 6-month & 1-month & \\
\hline \multicolumn{8}{|l|}{ South Africa } \\
\hline $\begin{array}{l}\text { Amare et al. } \\
\text { (2018) [67]. } \\
\text { Ethiopia }\end{array}$ & Suicide attempt & $\begin{array}{l}\text { School } \\
\text { (573) }\end{array}$ & $\begin{array}{l}\text { OV }=93 / 573 \\
(16.2 \%) \\
F=44 / 296 \\
(14.8 \%) \\
M=49 / 277 \\
(17.7 \%)\end{array}$ & - & - & $\begin{array}{l}\text { OV }=18 / 573 \\
(3.1 \%)\end{array}$ & $5 / 5$ \\
\hline $\begin{array}{l}\text { Khuzwayo et al. } \\
\text { (2018) [68]. } \\
\text { South Africa }\end{array}$ & Suicide attempt & $\begin{array}{l}\text { School } \\
(1687)\end{array}$ & - & $\begin{array}{l}\text { OV }=256 / 1687 \\
(15.2 \%) \\
F=196 / 854 \\
(22.9 \%) \\
M=60 / 833(7.2 \%)\end{array}$ & - & - & $3 / 5$ \\
\hline $\begin{array}{l}\text { Liu et al. } \\
\text { (2018) [69]. } \\
\text { Cross-national (Benin, } \\
\text { Ghana, Malawi, } \\
\text { Mauritania, Namibia, \& } \\
\text { eSwatini). }\end{array}$ & Suicide attempt & $\begin{array}{l}\text { School: } \\
\text { Benin } \\
(2649) \\
\text { Ghana } \\
\text { (3543) } \\
\text { Malawi } \\
\text { (2212) } \\
\text { Mauritania } \\
\text { (1976) } \\
\text { Namibia } \\
\text { (4410) } \\
\text { eSwatini } \\
\text { (3612) }\end{array}$ & - & 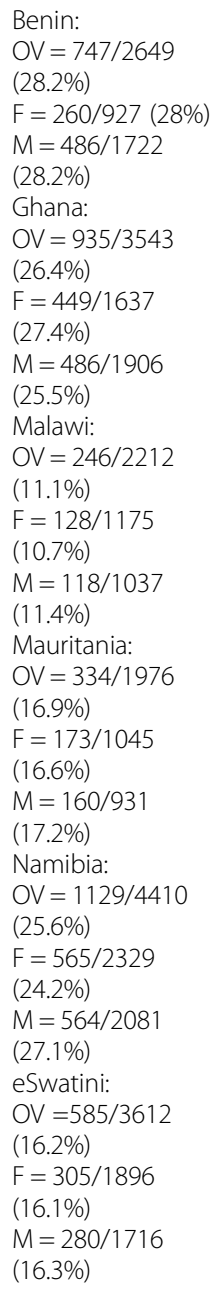 & - & - & $5 / 5$ \\
\hline $\begin{array}{l}\text { van der Wal \& George } \\
\text { (2018) [70]. } \\
\text { South Africa. }\end{array}$ & Self-harm & $\begin{array}{l}\text { School } \\
\text { (962) }\end{array}$ & $\begin{array}{l}\text { OV }=167 / 962 \\
(17.4 \%) \\
F=109 / 557 \\
(19.4 \%) \\
M=58 / 405 \\
(14.5 \%)\end{array}$ & - & - & - & $3 / 5$ \\
\hline $\begin{array}{l}\text { Baiden et al. } \\
\text { (2019) [71]. } \\
\text { Ghana }\end{array}$ & Suicide attempt & $\begin{array}{l}\text { School } \\
\text { (1633) }\end{array}$ & - & $\begin{array}{l}\text { OV }=349 / 1633 \\
(21.1 \%) \\
F=187 / 807 \\
(23.2 \%)\end{array}$ & - & - & $5 / 5$ \\
\hline
\end{tabular}


Table 2 Prevalence Estimates of Self-harm (by year and country of publication) (Continued)

\begin{tabular}{|c|c|c|c|c|c|c|c|}
\hline \multirow{3}{*}{$\begin{array}{l}\text { Author } \\
\text { (year) } \\
\text { Country }\end{array}$} & \multirow[t]{3}{*}{ Term } & \multirow[t]{3}{*}{$\begin{array}{l}\text { Setting } \\
\text { (sample) }\end{array}$} & \multicolumn{4}{|l|}{$\begin{array}{l}\text { Prevalence } \\
\text { estimate }\end{array}$} & \multirow[t]{2}{*}{$\begin{array}{l}\text { Study } \\
\text { quality }\end{array}$} \\
\hline & & & Lifetime & 12-month & 6-month & 1-month & \\
\hline & & & & $\begin{array}{l}M=162 / 826 \\
(19.6 \%)\end{array}$ & & & \\
\hline $\begin{array}{l}\text { Brittain et al. } \\
\text { (2019) [72]. } \\
\text { South Africa }\end{array}$ & $\begin{array}{l}\text { Self-harm } \\
\& \\
\text { Attempted suicide }\end{array}$ & $\begin{array}{l}\text { Community } \\
\text { (110) }\end{array}$ & - & $\begin{array}{l}\text { Self-harm: } \\
3 / 110(3 \%) \\
\text { Attempted } \\
\text { suicide: } \\
1 / 110(0.9 \%)\end{array}$ & - & - & $4 / 5$ \\
\hline $\begin{array}{l}\text { Darré et al. } \\
\text { (2019) [73]. } \\
\text { Togo }\end{array}$ & Suicide attempt & $\begin{array}{l}\text { School } \\
(941)\end{array}$ & $\begin{array}{l}\mathrm{OV}=46 / 941 \\
(4.9 \%) \\
F=31 / 753(3.3 \%) \\
M=15 / 188 \\
(1.6 \%)\end{array}$ & - & - & - & $3 / 5$ \\
\hline $\begin{array}{l}\text { Koyanagi, Oh et al. } \\
\text { (2019) [74]. } \\
\text { Cross-national (Benin, } \\
\text { Ghana, Malawi, } \\
\text { Mauritania, Mozambique, } \\
\text { Namibia, Seychelles, } \\
\text { Swaziland, \& Tanzania). }\end{array}$ & Suicide attempt & $\begin{array}{l}\text { School: } \\
\text { Benin } \\
(1170) \\
\text { Ghana } \\
(1110) \\
\text { Malawi } \\
\text { (2224) } \\
\text { Mauritania } \\
\text { (1285) } \\
\text { Mozambique } \\
\text { (668) } \\
\text { Namibia } \\
\text { (1936) } \\
\text { Seychelles } \\
\text { (2061) } \\
\text { Swaziland } \\
\text { (1318) } \\
\text { Tanzania } \\
\text { (2615) }\end{array}$ & - & $\begin{array}{l}\text { Benin: } \\
\text { OV=337/1170 } \\
(28.8 \%) \\
F=104 / 397 \\
(26.4 \%) \\
M=233 / 773 \\
(30.1 \%) \\
\text { Ghana: } \\
\text { OV=295/1110 } \\
(26.6 \%) \\
F=154 / 565 \\
(27.5 \%) \\
M=141 / 545 \\
(25.9 \%) \\
\text { Malawi: } \\
\text { OV=249/2224 } \\
(11.2 \%) \\
F=115 / 1079 \\
(10.7 \%) \\
M=128 / 1145 \\
(11.2 \%) \\
\text { Mauritania: } \\
\text { OV=227/1285 } \\
(17.7 \%) \\
F=98 / 601(16.3 \%) \\
M=126 / 684 \\
(18.4 \%) \\
\text { Mozambique: } \\
\text { OV=112/668 } \\
(16.8 \%) \\
F=56 / 337 \text { (16.7\%) } \\
M=57 / 331 \\
(17.1 \%)\end{array}$ & - & - & $5 / 5$ \\
\hline $\begin{array}{l}\text { Koyanagi, Oh et al. } \\
\text { (2019) [74]. }\end{array}$ & Suicide attempt & & - & $\begin{array}{l}\text { Namibia: } \\
\text { OV =507/1936 } \\
(26.2 \%) \\
F=260 / 1106 \\
(23.5 \%) \\
M=247 / 830 \\
(29.7 \%) \\
\text { Seychelles: } \\
\text { OV=435/2061 } \\
(21.1 \%) \\
F=215 / 1041 \\
(20.7 \%) \\
M=220 / 1020 \\
(21.6 \%) \\
\text { Swaziland: } \\
\text { OV = 202/1318 } \\
(15.3 \%) \\
F=121 / 803 \\
(15.1 \%)\end{array}$ & - & - & $5 / 5$ \\
\hline
\end{tabular}


Table 2 Prevalence Estimates of Self-harm (by year and country of publication) (Continued)

\begin{tabular}{|c|c|c|c|c|c|c|c|}
\hline \multirow{2}{*}{$\begin{array}{l}\text { Author } \\
\text { (year) } \\
\text { Country }\end{array}$} & \multirow[t]{2}{*}{ Term } & \multirow[t]{2}{*}{$\begin{array}{l}\text { Setting } \\
\text { (sample) }\end{array}$} & \multicolumn{4}{|l|}{$\begin{array}{l}\text { Prevalence } \\
\text { estimate }\end{array}$} & \multirow[t]{2}{*}{$\begin{array}{l}\text { Study } \\
\text { quality }\end{array}$} \\
\hline & & & Lifetime & 12-month & 6-month & 1-month & \\
\hline & & & & $\begin{array}{l}M=78 / 515 \\
(15.2 \%) \\
\text { Tanzania: } \\
\text { OV=290/2615 } \\
(11.1 \%) \\
F=159 / 1391 \\
(11.4 \%) \\
M=126 / 1224 \\
(10.3 \%)\end{array}$ & & & \\
\hline $\begin{array}{l}\text { Koyanagi, Stubbs et al. } \\
\text { (2019) [75]. } \\
\text { Cross-national (including } \\
\text { Benin, Ghana, Mauritania, } \\
\text { Mozambique, Namibia, } \\
\text { Seychelles, Swaziland, \& } \\
\text { Tanzania). }\end{array}$ & Suicide attempt & $\begin{array}{l}\text { School: } \\
\text { Benin (2690) } \\
\text { Ghana } \\
\text { (1684) } \\
\text { Mauritania } \\
\text { (2063) } \\
\text { Mozambique } \\
\text { (1918) } \\
\text { Namibia } \\
\text { (4531) } \\
\text { Seychelles } \\
\text { (2540) } \\
\text { Swaziland } \\
\text { (3680) } \\
\text { Tanzania } \\
\text { (3793) }\end{array}$ & - & $\begin{array}{l}\text { Benin: } \\
\text { 761/2690 (28.3\%) } \\
\text { Ghana: } \\
\text { 485/1684 (28.8\%) } \\
\text { Mauritania: } \\
\text { 359/2063 (17.4\%) } \\
\text { Mozambique: } \\
\text { 363/1918 (18.9\%) } \\
\text { Namibia: } \\
\text { 1178/4531 (26.0\%) } \\
\text { Seychelles: } \\
\text { 511/2540 (20.1\%) } \\
\text { Swaziland: } \\
\text { 607/3680 (16.5\%) } \\
\text { Tanzania: } \\
\text { 436/3793 (11.5\%) }\end{array}$ & - & - & $5 / 5$ \\
\hline $\begin{array}{l}\text { Nguyen et al. } \\
\text { (2019) [76]. } \\
\text { Cross-national (Nigeria, } \\
\text { Uganda, \& Zambia). }\end{array}$ & Self-injury & $\begin{array}{l}\text { Households: } \\
\text { Nigeria } \\
\text { (4203) } \\
\text { Uganda } \\
\text { (5804) } \\
\text { Zambia } \\
(1819)\end{array}$ & $\begin{array}{l}\text { Nigeria: } \\
\text { 123/4203 (2.9\%) } \\
\text { Uganda: } \\
\text { 244/5804 (4.2\%) } \\
\text { Zambia: } \\
\text { 104/1819 (5.7\%) }\end{array}$ & - & - & - & $5 / 5$ \\
\hline $\begin{array}{l}\text { Quarshie et al. } \\
\text { (2019) [77]. } \\
\text { Ghana }\end{array}$ & Suicide attempt & $\begin{array}{l}\text { College } \\
(305)\end{array}$ & $\begin{array}{l}\text { OV }=7 / 305 \\
(2.3 \%) \\
F=6 / 277(2.3 \%) \\
M=1 / 28(3.6 \%)\end{array}$ & - & - & - & $5 / 5$ \\
\hline $\begin{array}{l}\text { Shayo \& Lawala } \\
\text { (2019) [78]. } \\
\text { Tanzania }\end{array}$ & Suicide attempt & $\begin{array}{l}\text { School } \\
\text { (3793) }\end{array}$ & - & $\begin{array}{l}\text { OV }=422 / 3793 \\
(11.1 \%) \\
F=230 / 1974 \\
(11.7 \%) \\
M=192 / 1819 \\
(10.6 \%)\end{array}$ & - & - & $5 / 5$ \\
\hline $\begin{array}{l}\text { Thornton et al. } \\
\text { (2019) [79]. } \\
\text { Cross-national (South } \\
\text { Africa \& Guyana) }\end{array}$ & Suicide attempt & $\begin{array}{l}\text { Households: } \\
\text { South Africa } \\
\text { (175) }\end{array}$ & - & - & $\begin{array}{l}\text { South Africa: } \\
\text { 14/175 (7.4\%) }\end{array}$ & - & $2 / 5$ \\
\hline $\begin{array}{l}\text { Tolulope et al. } \\
\text { (2019) [80]. } \\
\text { Nigeria. }\end{array}$ & Suicide attempt & $\begin{array}{l}\text { School } \\
(1015)\end{array}$ & $\begin{array}{l}\mathrm{OV}=30 / 1015 \\
(3 \%)\end{array}$ & - & - & - & $4 / 5$ \\
\hline $\begin{array}{l}\text { Uddin et al. } \\
\text { (2019) [81]. } \\
\text { Cross-national (Benin, } \\
\text { Ghana, Malawi, } \\
\text { Mauritania, Mozambique, } \\
\text { Namibia, Swaziland, \& } \\
\text { Tanzania). }\end{array}$ & Suicide attempt & $\begin{array}{l}\text { School: } \\
\text { Benin } \\
(2579) \\
\text { Ghana } \\
(2195) \\
\text { Malawi } \\
\text { (2191) } \\
\text { Mauritania } \\
\text { (1867) } \\
\text { Mozambique } \\
\text { (1248) }\end{array}$ & - & $\begin{array}{l}\text { Benin: } \\
\text { OV =720/2579 } \\
(27.9 \%) \\
F=254 / 902 \\
(28.2 \%) \\
M=466 / 1667 \\
(28 \%) \\
\text { Ghana: } \\
\text { OV=607/2195 } \\
(27.7 \%) \\
F=304 / 1029 \\
(29.5 \%) \\
M=294 / 1146\end{array}$ & - & - & $5 / 5$ \\
\hline
\end{tabular}


Table 2 Prevalence Estimates of Self-harm (by year and country of publication) (Continued)

\begin{tabular}{|c|c|c|c|c|c|c|c|}
\hline \multirow{2}{*}{$\begin{array}{l}\text { Author } \\
\text { (year) } \\
\text { Country }\end{array}$} & \multirow[t]{2}{*}{ Term } & \multirow[t]{2}{*}{$\begin{array}{l}\text { Setting } \\
\text { (sample) }\end{array}$} & \multicolumn{4}{|c|}{$\begin{array}{l}\text { Prevalence } \\
\text { estimate }\end{array}$} & \multirow[t]{2}{*}{$\begin{array}{l}\text { Study } \\
\text { quality }\end{array}$} \\
\hline & & & Lifetime & 12-month & 6-month & 1-month & \\
\hline & & & & $\begin{array}{l}(25.7 \%) \\
\text { Malawi: } \\
\text { OV=251/2191 } \\
(11.5 \%) \\
F=125 / 1142 \\
(11 \%) \\
M=118 / 1015 \\
(11.6 \%)\end{array}$ & & & \\
\hline $\begin{array}{l}\text { Uddin et al. } \\
\text { (2019) [81]. }\end{array}$ & Suicide attempt & $\begin{array}{l}\text { Namibia } \\
\text { (3235) } \\
\text { Swaziland } \\
\text { (2547) } \\
\text { Tanzania } \\
\text { (2911) }\end{array}$ & - & $\begin{array}{l}\text { Mauritania: } \\
\text { OV=322/1867 } \\
(17.3 \%) \\
\mathrm{F}=161 / 983 \\
(16.4 \%) \\
\mathrm{M}=153 / 866 \\
(17.7 \%) \\
\text { Mozambique: } \\
\text { OV=213/1248 } \\
(17.1 \%) \\
\mathrm{F}=113 / 596(19 \%) \\
\mathrm{M}=95 / 633(15 \%) \\
\text { Namibia: } \\
\text { OV=836/3235 } \\
(25.8 \%) \\
\mathrm{F}=432 / 1795 \\
(24.1 \%) \\
\mathrm{M}=390 / 1409 \\
(27.7 \%) \\
\mathrm{Swaziland} \\
\text { OV=439/2547 } \\
(17.2 \%) \\
\mathrm{F}=245 / 1432 \\
(17.1 \%) \\
\mathrm{M}=190 / 1108 \\
(17.2 \%) \\
\text { Tanzania: } \\
\text { OV=298/2911 } \\
(10.2 \%) \\
\mathrm{F}=161 / 1448 \\
(10.9 \%) \\
\mathrm{M}=126 / 1403 \\
(9 \%)\end{array}$ & - & - & $5 / 5$ \\
\hline $\begin{array}{l}\text { Vancampfort et al. } \\
\text { (2019) [82]. } \\
\text { Cross-national (Benin, } \\
\text { Ghana, Mauritania, } \\
\text { Mozambique, Namibia, } \\
\text { Seychelles, \& Tanzania) }\end{array}$ & Suicide attempt & $\begin{array}{l}\text { School: } \\
\text { Benin } \\
(1170) \\
\text { Ghana } \\
\text { (1110) } \\
\text { Mauritania } \\
\text { (1285) } \\
\text { Mozambique } \\
\text { (668) } \\
\text { Namibia } \\
\text { (1936) }\end{array}$ & - & $\begin{array}{l}\text { Benin: } \\
\text { 323/1170 (27.6\%) } \\
\text { Ghana: } \\
\text { 295/1110 (26.6\%) } \\
\text { Mauritania: } \\
\text { 213/1285 (16.6\%) } \\
\text { Mozambique: } \\
\text { 114/668 (16.5\%) } \\
\text { Namibia: } \\
\text { 492/1936 (25.4\%) }\end{array}$ & - & - & $5 / 5$ \\
\hline $\begin{array}{l}\text { Vancampfort et al. } \\
\text { (2019) [82]. }\end{array}$ & Suicide attempt & $\begin{array}{l}\text { Seychelles } \\
(2061) \\
\text { Tanzania } \\
(2615)\end{array}$ & - & $\begin{array}{l}\text { Seychelles: } \\
\text { 423/2061 (20.5\%) } \\
\text { Tanzania: } \\
\text { 282/2615 (10.8\%) }\end{array}$ & - & - & $5 / 5$ \\
\hline
\end{tabular}

OV Overall estimate

F Female

$M$ Male

SAYRBS South African Youth Risk Behaviour Survey

Gage's (2013) [25] reported prevalence period was 3 months. Van Rooyen's (2013) [50] reported prevalence period was 11 months 
Tanzania, and Uganda). Seventy-two of the studies were in English and two were in French.

The majority of the studies $(n=56 ; 75.6 \%)$ utilised a quantitative cross-sectional design involving questionnaires administered to participants accessed in communities/households, a charity facility, hospitals, schools and universities (see Additional file 6). Five studies included only female participants [22-26]; and one study involved only male participants [27]. The majority $(n=$ 49; $66.2 \%$ ) of the studies sampled students from schools and universities. Six studies (8.1\%) sampled young people who were out of the school environment, including adolescents living in poor, rural, war-affected communities [28], adolescents in children's homes [29], children and youth living in slums and streets [30, 31], adolescents living in poor urban and rural villages [32], and out-of-school youth who were unstably housed, living in poor urban neighbourhoods [33].

The total sample covered by the studies was 205,132. Thirty-nine $(52.7 \%)$ of the studies used some form of random selection in recruiting their participants. Across the 74 studies, only $14(18.9 \%)$ provided information on the size of their target population and how their sample sizes were determined.

Six $(8.1 \%)$ of the studies were rated "average quality" on the MMAT, 21 studies (28.4\%) were rated "above average quality"; 22 studies (29.7\%) were rated "high quality", while the remaining 25 studies (33.8\%) were rated to be of "very high quality" (see Additional file 7).

\section{Main findings}

Fifty-five (74.3\%) of the studies reported prevalence estimates, though none reported lifetime, 12-month, 6month, and 1-month estimates, with 12-month estimates favoured by the majority $(n=30 ; 54.5 \%)$. As shown in Table 2, the reported lifetime prevalence estimates ranged from $1.4 \%$ to $48.3 \%$ [28, 50]; the 12 -month prevalence estimates varied between $0.9 \%$ and $35.8 \%$ [52, 72]; the reported 6-month prevalence ranged from $7 \cdot 4 \%$ to $22.7 \%[55,79]$; and the 1-month reported prevalence estimates varied between $1.9 \%$ and $26.4 \%$ [31, 58].

The majority of the 55 prevalence studies $(n=46$; $83.6 \%$ ) focused on suicidal self-harm, seven studies $(12.7 \%)$ focused on non-suicidal self-harm [48, 50, 52, $53,60,70,76]$, while two studies simultaneously reported the prevalence estimates of both suicidal selfharm (suicidal attempt) and non-suicidal self-harm (selfinjury) $[28,72]$.

Visual inspection of the forest plots and the $\mathrm{I}^{2}$ value ranges $(98.84 \%$ to $99.71 \%, \mathrm{p}<\cdot 001)$ indicate that heterogeneity across each of these summaries is substantial (see Figs. 1, 2, 3 and 4). Median values with interquartile ranges were computed for the overall and sub-regional reported prevalence estimates (see Fig. 5). Considerable variability was found across the ranges of prevalence estimates reported: the median lifetime prevalence estimate was $10.3 \%$ (interquartile range [IQR] of $4.6 \%-16.1 \%$ ) and the median 12 month prevalence estimate was $16.9 \%$ (IQR: $11.5 \%$

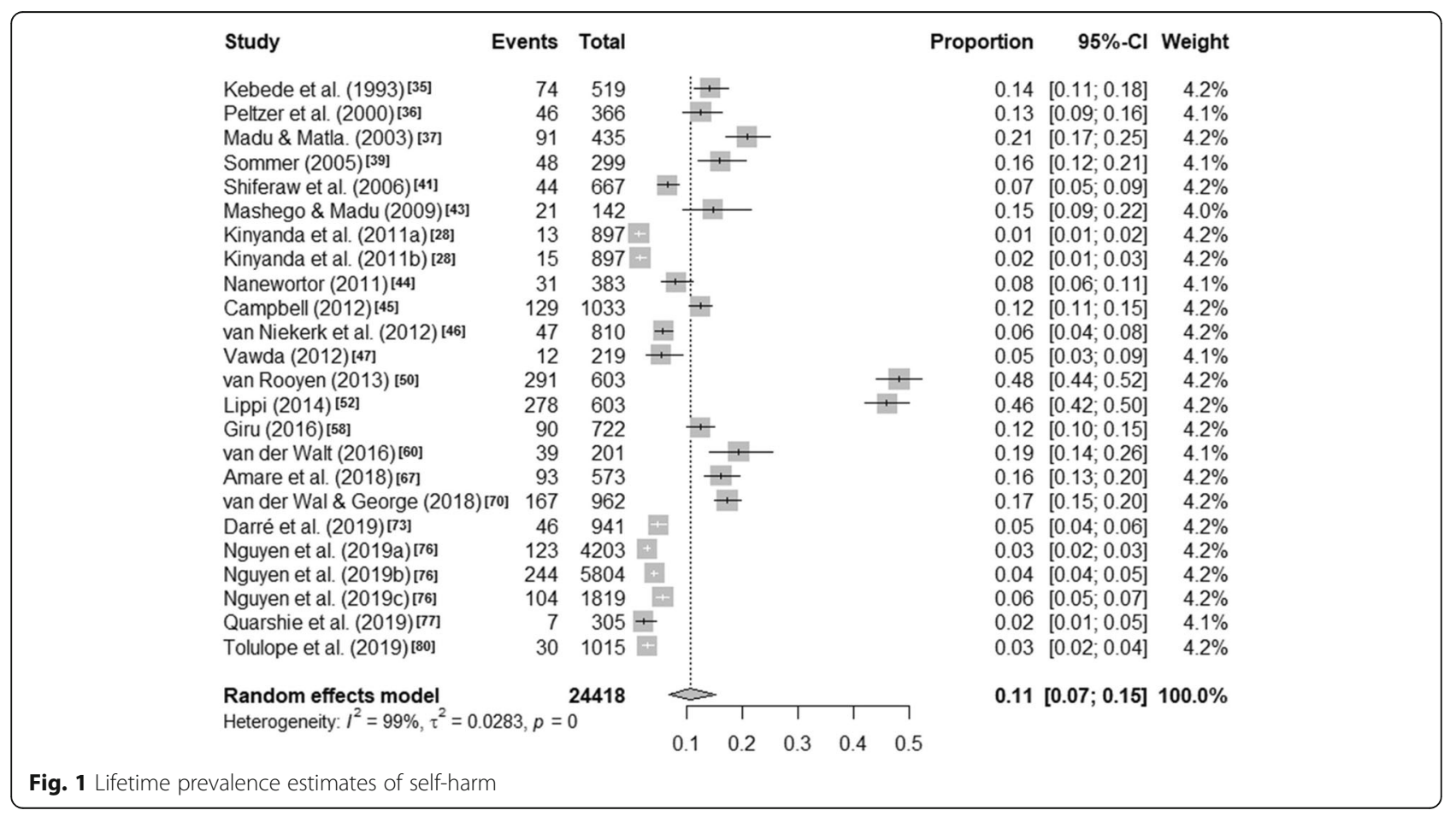




\begin{tabular}{|c|c|c|c|c|c|c|c|c|c|}
\hline & Study & Events & Total & & & & Proportion & $95 \%-\mathrm{Cl}$ & Weight \\
\hline & Flisher et al. (1993) [34] & 573 & 7340 & + & & & 0.08 & {$[0.07 ; 0.08]$} & $1.6 \%$ \\
\hline & Wild et al. (2004) [38] & 95 & 939 & + & & & 0.10 & {$[0.08 ; 0.12]$} & $1.6 \%$ \\
\hline & Flisher et al. (2006) [40] & 971 & 10669 & + & & & 0.09 & {$[0.09 ; 0.10]$} & $1.6 \%$ \\
\hline & Shiferaw et al. (2006)[41] & 39 & 667 & $\mp$ & & & 0.06 & {$[0.04 ; 0.08]$} & $1.6 \%$ \\
\hline & Omigbodun et al. (2008) [20] & 167 & 1429 & + & & & 0.12 & {$[0.10 ; 0.13]$} & $1.6 \%$ \\
\hline & Peltzer (2008) [42] & 278 & 1157 & & & + & 0.24 & {$[0.22 ; 0.27]$} & $1.6 \%$ \\
\hline & Swahn et al. (2012)[30] & 90 & 457 & & & & 0.20 & {$[0.16 ; 0.24]$} & $1.5 \%$ \\
\hline & Muula et al. (2013)[48] & 254 & 2136 & $\mp$ & & & 0.12 & {$[0.11 ; 0.13]$} & $1.6 \%$ \\
\hline & Cheng et al. (2014a) [33] & 73 & 449 & $\rightarrow$ & & & 0.16 & {$[0.13 ; 0.20]$} & $1.5 \%$ \\
\hline & Cheng et al. (2014b) [33] & 54 & 496 & $\rightarrow$ & & & 0.11 & {$[0.08 ; 0.14]$} & $1.6 \%$ \\
\hline & Chinawa et al. (2014)[51] & 96 & 764 & + & & & 0.13 & {$[0.10 ; 0.15]$} & $1.6 \%$ \\
\hline & Lippi (2014) [52] & 216 & 603 & & & $\longrightarrow$ & 0.36 & {$[0.32 ; 0.40]$} & $1.6 \%$ \\
\hline & Penning \& Collings (2014) [53] & 20 & 716 & $\mp$ & & & 0.03 & {$[0.02 ; 0.04]$} & $1.6 \%$ \\
\hline & Randall et al. (2014) [54] & 761 & 2690 & & & + & 0.28 & {$[0.27 ; 0.30]$} & $1.6 \%$ \\
\hline & Shaikh et al. (2016) [59] & 287 & 2225 & + & & & 0.13 & {$[0.12 ; 0.14]$} & $1.6 \%$ \\
\hline & Akanni et al. (2017) [61] & 17 & 300 & $\mp$ & & & 0.06 & {$[0.03 ; 0.09]$} & $1.5 \%$ \\
\hline & Asante et al. (2017)[62] & 440 & 1984 & & + & + & 0.22 & {$[0.20 ; 0.24]$} & $1.6 \%$ \\
\hline & Nyandindi $(2017)^{[64]}$ & 436 & 3793 & + & & & 0.11 & {$[0.10 ; 0.13]$} & $1.6 \%$ \\
\hline & Peltzer \& Pengpid (2017) [65] & 1029 & 4531 & & + & + & 0.23 & {$[0.21 ; 0.24]$} & $1.6 \%$ \\
\hline & Stansfeld et al. (2017) [66] & 139 & 1034 & + & & & 0.13 & {$[0.11 ; 0.16]$} & $1.6 \%$ \\
\hline & Baiden et al. (2019) $)^{[71]}$ & 345 & 1633 & & + & & 0.21 & {$[0.19 ; 0.23]$} & $1.6 \%$ \\
\hline & Khuzwayo et al. (2018) [68] & 267 & 1759 & + & & & 0.15 & {$[0.14 ; 0.17]$} & $1.6 \%$ \\
\hline & Liu et al. (2018a)[69] & 747 & 2649 & & & + & 0.28 & {$[0.26 ; 0.30]$} & $1.6 \%$ \\
\hline & Liu et al. (2018b)[69] & 935 & 3543 & & & + & 0.26 & {$[0.25 ; 0.28]$} & $1.6 \%$ \\
\hline & Liu et al. (2018c) [69] & 246 & 2212 & + & & & 0.11 & {$[0.10 ; 0.13]$} & $1.6 \%$ \\
\hline & Liu et al. (2018d)[69] & 334 & 1976 & $t$ & + & & 0.17 & {$[0.15 ; 0.19]$} & $1.6 \%$ \\
\hline & Liu et al. $(2018 \mathrm{e})$ [69] & 1129 & 4410 & & & + & 0.26 & {$[0.24 ; 0.27]$} & $1.6 \%$ \\
\hline & Liu et al. $(2018 f)[69]$ & 585 & 3612 & + & & & 0.16 & {$[0.15 ; 0.17]$} & $1.6 \%$ \\
\hline & Brittain et al. (2019a) [72] & 3 & $110+$ & + & & & 0.03 & {$[0.01 ; 0.08]$} & $1.4 \%$ \\
\hline & Brittain et al. (2019b) [72] & 1 & $110+$ & + & & & 0.01 & {$[0.00 ; 0.05]$} & $1.4 \%$ \\
\hline & Koyanagi, Oh et al (2019a) [74] & 337 & 1170 & & & + & 0.29 & {$[0.26 ; 0.31]$} & $1.6 \%$ \\
\hline & Koyanagi, Oh et al (2019b) 774 & 295 & 1110 & & & $\mp$ & 0.27 & {$[0.24 ; 0.29]$} & $1.6 \%$ \\
\hline & Koyanagi, Oh et al (2019c) [74] & 249 & 2224 & + & & & 0.11 & {$[0.10 ; 0.13]$} & $1.6 \%$ \\
\hline & Koyanagi, Oh et al (2019d)[74] & 227 & 1285 & & ; & & 0.18 & {$[0.16 ; 0.20]$} & $1.6 \%$ \\
\hline & Koyanagi, Oh et al $(2019 \mathrm{e}){ }^{[74]}$ & 112 & 668 & - & & & 0.17 & {$[0.14 ; 0.20]$} & $1.6 \%$ \\
\hline & Koyanagi, Oh et al (2019f) [74] & 507 & 1936 & & & + & 0.26 & {$[0.24 ; 0.28]$} & $1.6 \%$ \\
\hline & Koyanagi, Oh et al $(2019 \mathrm{~g})[74]$ & 435 & 2061 & & + & & 0.21 & {$[0.19 ; 0.23]$} & $1.6 \%$ \\
\hline & Koyanagi, Oh et al $(2019 \mathrm{~h})[74]$ & 202 & 1318 & + & & & 0.15 & {$[0.13 ; 0.17]$} & $1.6 \%$ \\
\hline & Koyanagi, Oh et al (2019i) [74] & 290 & 2615 & +1 & & & 0.11 & {$[0.10 ; 0.12]$} & $1.6 \%$ \\
\hline & Koyanagi, Stubbs et al (2019a) [75] & 5] 761 & 2690 & & & + & 0.28 & {$[0.27 ; 0.30]$} & $1.6 \%$ \\
\hline & Koyanagi, Stubbs et al (2019b) [75] & 5] 485 & 1684 & & & + & 0.29 & {$[0.27 ; 0.31]$} & $1.6 \%$ \\
\hline & Koyanagi, Stubbs et al (2019c) [75] & (5] 359 & 2063 & & i & & 0.17 & {$[0.16 ; 0.19]$} & $1.6 \%$ \\
\hline & Koyanagi, Stubbs et al (2019d) [75] & 5] 363 & 1918 & & + & & 0.19 & {$[0.17 ; 0.21]$} & $1.6 \%$ \\
\hline & Koyanagi, Stubbs et al (2019e) $[75]$ & 5] 1178 & 4531 & & & \pm & 0.26 & {$[0.25 ; 0.27]$} & $1.6 \%$ \\
\hline & Koyanagi, Stubbs et al (2019f) [75] & 5] 511 & 2540 & & + & & 0.20 & {$[0.19 ; 0.22]$} & $1.6 \%$ \\
\hline & Koyanagi, Stubbs et al (2019g)[75] & (5] 607 & 3680 & 랄 & & & 0.16 & {$[0.15 ; 0.18]$} & $1.6 \%$ \\
\hline & Koyanagi, Stubbs et al $(2019 \mathrm{~h})[75]$ & (5] 436 & 3793 & \pm & & & 0.11 & {$[0.10 ; 0.13]$} & $1.6 \%$ \\
\hline & Shayo \& Lawala (2019) [78] & 415 & 3793 & + & & & 0.11 & {$[0.10 ; 0.12]$} & $1.6 \%$ \\
\hline & Uddin et al. (2019a)[81] & 720 & 2579 & & & + & 0.28 & {$[0.26 ; 0.30]$} & $1.6 \%$ \\
\hline & Uddin et al. (2019b) [81] & 608 & 2195 & & & + & 0.28 & {$[0.26 ; 0.30]$} & $1.6 \%$ \\
\hline & Uddin et al. (2019c) [81] & 252 & 2191 & $\mp$ & & & 0.12 & {$[0.10 ; 0.13]$} & $1.6 \%$ \\
\hline & Uddin et al. (2019d) [81] & 323 & 1867 & & $\vdots$ & & 0.17 & {$[0.16 ; 0.19]$} & $1.6 \%$ \\
\hline & Uddin et al. (2019e)[81] & 213 & 1248 & & $\because$ & & 0.17 & {$[0.15 ; 0.19]$} & $1.6 \%$ \\
\hline & Uddin et al. (2019f)[81] & 835 & 3235 & & & $\mp$ & 0.26 & {$[0.24 ; 0.27]$} & $1.6 \%$ \\
\hline & Uddin et al. (2019g)[81] & 438 & 2547 & & + & & 0.17 & {$[0.16 ; 0.19]$} & $1.6 \%$ \\
\hline & Uddin et al. (2019h) [81] & 297 & 2911 & + & & & 0.10 & {$[0.09 ; 0.11]$} & $1.6 \%$ \\
\hline & Vancampfort et al. (2019a) [82] & 323 & 1170 & & & + & 0.28 & {$[0.25 ; 0.30]$} & $1.6 \%$ \\
\hline & Vancampfort et al. (2019b)[82] & 295 & 1110 & & & + & 0.27 & {$[0.24 ; 0.29]$} & $1.6 \%$ \\
\hline & Vancampfort et al. (2019c) [82] & 213 & 1285 & $\rightarrow$ & & & 0.17 & {$[0.15 ; 0.19]$} & $1.6 \%$ \\
\hline & Vancampfort et al. (2019d) [82] & 114 & 668 & - & $\div$ & & 0.17 & {$[0.14 ; 0.20]$} & $1.6 \%$ \\
\hline & Vancampfort et al. $(2019 e)[82]$ & 492 & 1936 & & & + & 0.25 & {$[0.23 ; 0.27]$} & $1.6 \%$ \\
\hline & Vancampfort et al. (2019f) ${ }^{[2]}$ & 423 & 2061 & & + & & 0.21 & {$[0.19 ; 0.22]$} & $1.6 \%$ \\
\hline & Vancampfort et al. (2019g) [82] & 282 & 2615 & F & & & 0.11 & {$[0.10 ; 0.12]$} & $1.6 \%$ \\
\hline & \multicolumn{3}{|c|}{ Random effects model $\quad 137090$} & & P & & \multirow{3}{*}{\multicolumn{3}{|c|}{$0.17[0.15 ; 0.19] 100.0 \%$}} \\
\hline & \multirow{2}{*}{\multicolumn{3}{|c|}{ Heterogeneity: $I^{2}=99 \%, \tau^{2}=0.0114, p=0$}} & & 1 & $\neg$ & & & \\
\hline & & & & 0.1 & 0.2 & 0.3 & & & \\
\hline
\end{tabular}

25.5\%). Studies from Western sub-Saharan Africa reported the highest 12-month prevalence estimates ( median $=24 \cdot 3 \% ;$ IQR $=16 \cdot 9 \%-27 \cdot 9 \%$ ), while studies from Eastern $($ median $=11.5 \% ; \quad I Q R=11.1 \%-18.3 \%)$ and Southern $($ median $=16.5 \% ; \mathrm{IQR}=10.9 \%-24.0 \%)$
sub-Saharan Africa reported relatively similar median 12-month prevalence estimates.

Twenty (27\%) of the 74 studies reported on the methods of self-harm (Table 3). Overdose of medication was frequently reported from clinic-based studies, while 


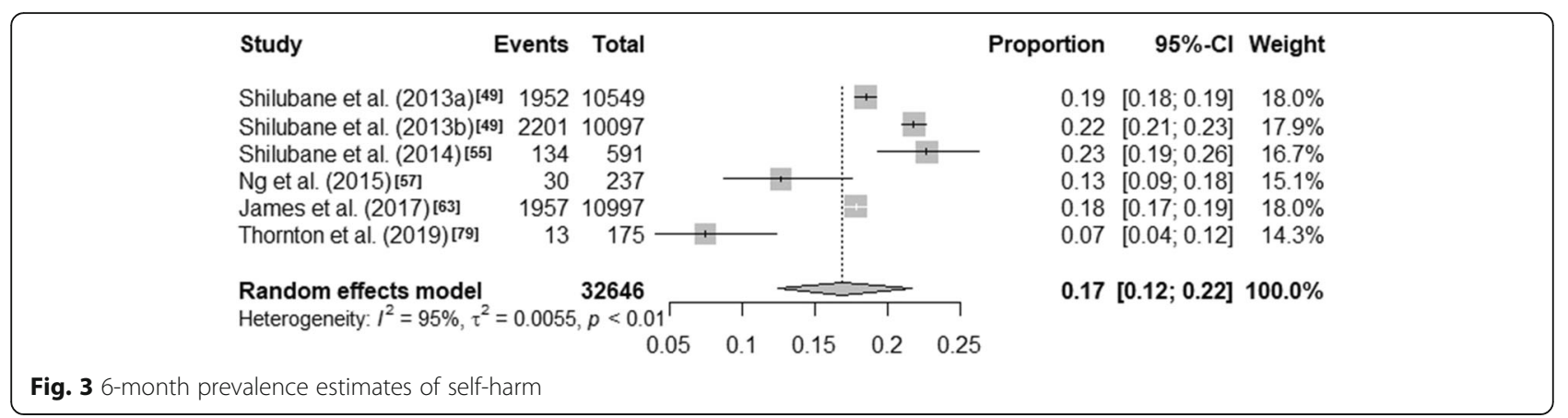

self-cutting was the predominant method reported in the non-clinic based studies.

Overall, 48 (64.9\%) of the 74 studies reported on the associates, risks and protective factors. The evidence was organised by reported associates into four main domains: personal, family, school, and interpersonal (nonfamily). The interpersonal (non-family) included circumstances related to the individuals' relationships with peers and neighbours, and other social relationships and interactions outside the family and school contexts. Additionally, based on the strong association of abuse and violence victimisation (within and outside the family context) and self-harm [11, 92-94], we created a separate category, abuse and violence, to capture all factors related to psychological, physical, emotional, and sexual abuse items.

Results are shown in Table 4. Common examples of associations included, at the personal level, depression, hopelessness and psychiatric illness; at the family level, conflict with parents, parental divorce; at school level, academic failure, and for the interpersonal level, relationship breakups and problems, and lack of social support. Abuse and violence-related factors included sexual abuse, dating violence, bullying, and physical fights. Only one study reported risk factors related to self-harm [56], while no study reported protective factors against self-harm.

Because of the substantial heterogeneity in samples, definition and measurement of associations, we regard any attempt at comparison or pooling of the reported prevalences of these associations (as opposed to simply their presence or absence) as potentially misleading. We noted, however, the proportion of included studies that reported associations in each category as follows: Personal $=41 / 48 ;$ Family $=31 / 48$; Interpersonal $=24 / 48$; Abuse and violence $=19 / 48$; School $=13 / 48$.

We further categorised the self-reported reasons for self-harm into "intrapersonal" (i.e., reasons intended to change one's state or circumstances), and "interpersonal" (i.e., reasons intended to change the state or circumstances of significant others). Eight (10.8\%) studies included self-reported reasons for self-harm - see Table 5 . Five of these were clinic-based, $[22-24,26,88]$ while three were non-clinic based [27, 29, 50].

Across the eight studies reporting reasons for selfharm, participants concurrently reported both intrapersonal and interpersonal reasons for engaging in selfharm with no clear pattern discernible.

The findings of the prevalence studies regarding associates of self-harm were mixed in terms of age, although more reported higher estimates among young people between the ages of 15 and 17 years, compared to those aged 14 years and below, and 18 years or above [35, 39, 43, 73].

The majority of the prevalence studies reported higher estimates among female adolescents than in male adolescents $[30,34,40,45,58,59,61,62,65,73,78]$, although seven studies $(12.7 \%)$ found higher prevalence estimates in male adolescents [33, 35, 37, 52, 55, 67, 77].

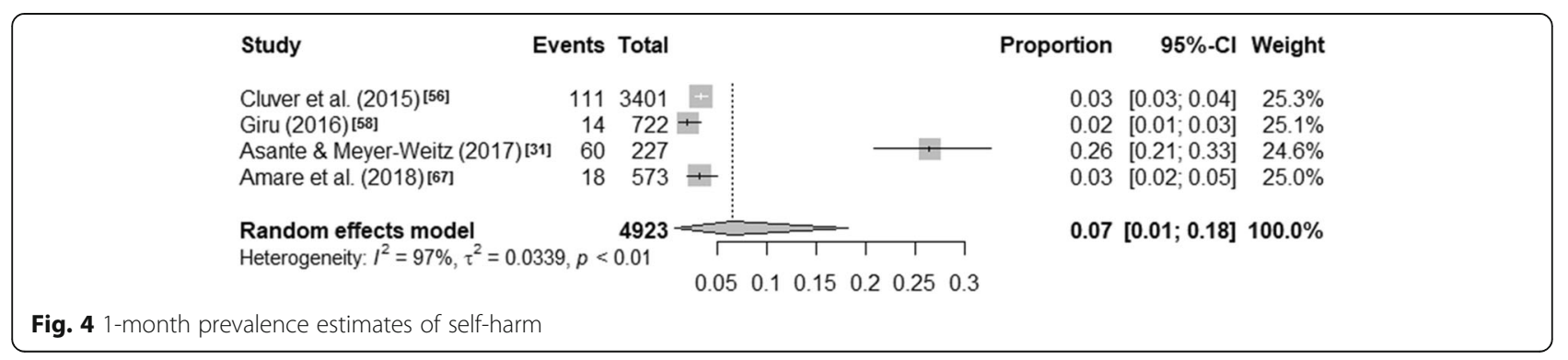




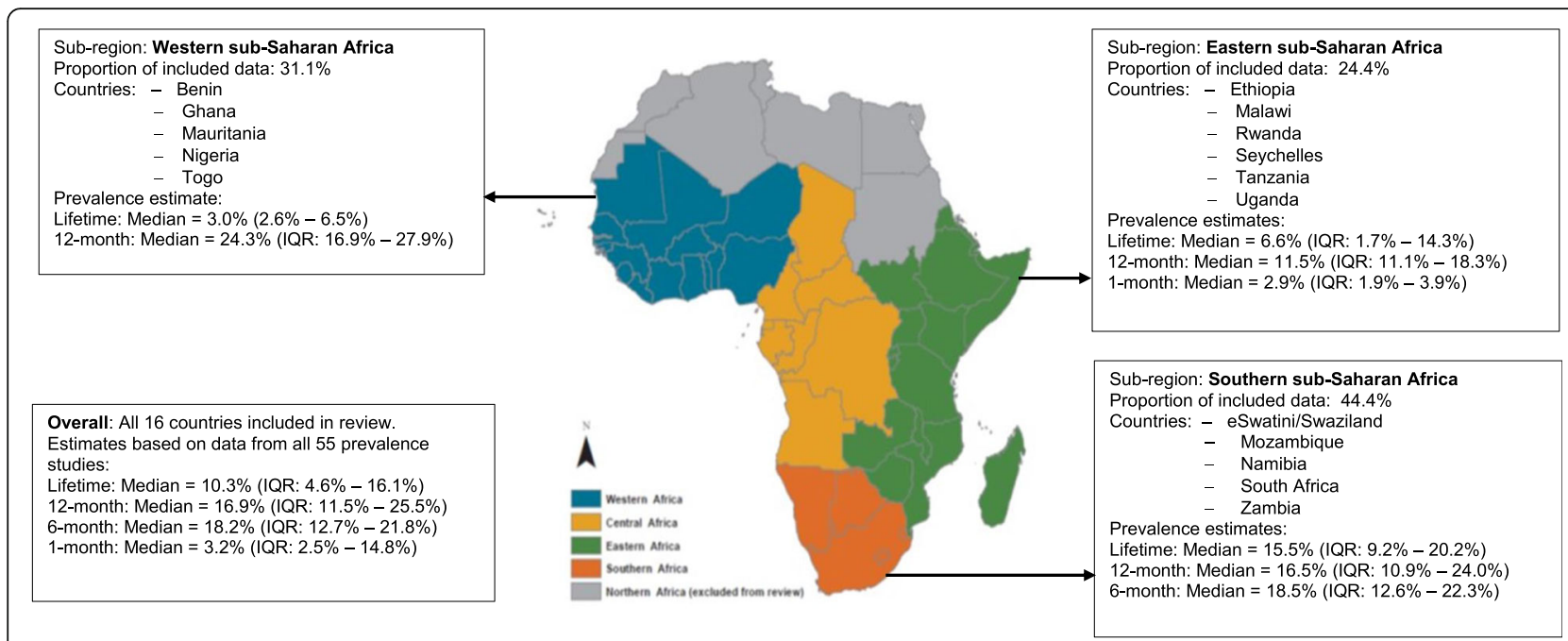

Fig. 5 Median and interquartile range (IQR) of prevalence estimates of adolescent self-harm in sub-Saharan Africa. Source: Map created by authors, based on the list of countries within the sub-regional division of sub-Saharan Africa by the United Nations Statistics Division's classification (list accessed on January 20, 2019: https://unstats.un.org/unsd/methodology/m49/)

\section{Discussion}

There are clearly problems with the literature we found. Despite the number of reported studies, and the extended search, we identified research from fewer than $40 \%(18 / 46)$ of the countries across the sub-region, and half of all studies came from one country - South Africa. This represents a serious gap in our knowledge about population mental health in those countries from which research is missing.

The reported prevalence estimates showed considerable variations within and between the countries and sub-regions of sub-Saharan Africa. Undoubtedly, real variations are likely to exist but there must also be methodological reasons to explain, for example, that estimates of lifetime prevalence appear lower than 12month prevalence. One explanation is the origin and contextual relevance of the measures used by the studies, and the lack of consistency in definitions and choice of measures across studies. In this respect it is worth noting the widespread use of the term "attempted suicide" in studies which did not explain the term to those responding to the question. The consequence is that studies cannot be reliably categorised as reporting the prevalence of suicidal or non-suicidal behaviour. For example - as shown on Table 5 respondents in a study about "attempted suicide" might report that they intended to die as the result of an act while others might report that the act was intended to prevent suicide, or that the act was designed to change the nature of their relationships with others. We found no substantial study where participants were asked in detail about reasons for self-harm, after responding with an initial affirmative to a question about attempted suicide. Respondent bias due to the illegality, sensitivity, stigma, and taboo against suicide and other self-destructive behaviours in Africa [98] is likely to vary according to how and by whom inquiries were made.

Even so, the median 12-month prevalence estimate of 16.9\% (IQR: $11.5 \%-25.5 \%$ ), and median 6-month prevalence estimate of $18.2 \%$ (IQR: $12.7 \%$ - 21.8\%) particularly had reasonably narrow confidence intervals. What is striking is that these figures are of the same order as those reported from Europe and North America, as is the finding that young women report more self-harm than young men. Similarly, methods of self-harm were similar with overdose of medication frequently reported in clinic-based studies, while self-cutting was the predominant method reported in the non-clinic based studies.

Generally, the studies reported multiple factors to be associated with self-harm at the personal level - sex, age, depression, hopelessness, psychiatric illness, alcohol and illicit drug use, at the family level - conflict with parents, at school-level - academic failure, bullying victimisation, truancy, and at the interpersonal level - breakup, sexual and physical abuse, romantic relationship problems, social support.

Various forms of abuse and violence victimisation occurring in the family, school, and interpersonal contexts were also reported. It was not possible to tell from all the studies we reviewed how often relationship problems in family, school, or social groups was marked by violence or abuse, but the circumstances of life in Ghana suggests the possibility of frequent exposure to such experiences. Relative to high-income countries, these circumstances are arguably likely to be more common in 
Table 3 Predominant Form / Method of Self-harm by year of publication

\begin{tabular}{|c|c|c|c|c|c|}
\hline \multirow{2}{*}{$\begin{array}{l}\text { Author } \\
\text { (year) } \\
\text { Country }\end{array}$} & \multirow[t]{2}{*}{ Setting } & \multirow{2}{*}{$\begin{array}{l}\text { Sample } \\
\text { (Sex) }\end{array}$} & \multicolumn{2}{|l|}{ Reported method of self-harm } & \multirow{2}{*}{$\begin{array}{l}\text { Study } \\
\text { quality }\end{array}$} \\
\hline & & & Self-Poisoning & Self-Injury & \\
\hline $\begin{array}{l}\text { Cummins \& Allwood } \\
\text { (1984) [83] } \\
\text { South Africa }\end{array}$ & $\begin{array}{l}\text { General } \\
\text { hospital }\end{array}$ & $\begin{array}{l}N=81 \\
(F=54, M=27)\end{array}$ & $\begin{array}{l}\text { - Overdose }=64 / 81(79 \%) \\
F=46 / 54(85.2 \%) \\
M=18 / 27(66.7 \%)\end{array}$ & - & $3 / 5$ \\
\hline $\begin{array}{l}\text { Schlebusch } \\
\text { (1985) [84] } \\
\text { South Africa }\end{array}$ & $\begin{array}{l}\text { General } \\
\text { hospital }\end{array}$ & $\begin{array}{l}N=159 \\
(F=115, M=44)\end{array}$ & $\begin{array}{l}\text { - Overdose, } 151 / 159(95 \%) \\
F=112 / 115(97.4 \%) \\
M=39 / 44(88.6 \%)\end{array}$ & $\begin{array}{l}\text { - Wrist/arm cutting = 8/159 (5\%) } \\
F=5 / 115(4.3 \%) \\
M=3 / 44(6.8 \%)\end{array}$ & $4 / 5$ \\
\hline $\begin{array}{l}\text { Pillay } \\
\text { (1987) [85] } \\
\text { South Africa }\end{array}$ & $\begin{array}{l}\text { General } \\
\text { hospital }\end{array}$ & $\begin{array}{l}N=55 \\
(F=42, M=13)\end{array}$ & - Overdose = 55/55 (100\%) & - & $3 / 5$ \\
\hline $\begin{array}{l}\text { Pillay } \\
\text { (1988) [86] } \\
\text { South Africa }\end{array}$ & $\begin{array}{l}\text { General } \\
\text { hospital }\end{array}$ & $\begin{array}{l}N=87 \\
(F=68, M=19)\end{array}$ & $\begin{array}{l}\text { - Self-poisoning = } 81 / 87(93.1 \%) \\
F=67 / 68(98.5 \%) \\
M=14 / 19(73.7 \%) \\
\text { - Carbon monoxide }=1 / 87(1.1 \%) \\
F=0 / 68 \\
M=1 / 19(5.3 \%)\end{array}$ & $\begin{array}{l}\text { - Wrist cutting = } 1 / 87(1.1 \%) \\
F=1 / 68(1.5 \%) \\
M=0 / 19 \\
\text { : Hanging = } 2 / 87(2.3 \%) \\
F=0 / 68 \\
M=2 / 19(10.5 \%) \\
\text { - Stabbing = } 1 / 87(1.1 \%) \\
F=0 / 68 \\
M=1 / 19(5.3 \%) \\
\text { - Jumping from height }=1 / 87(1.1 \%) \\
F=0 / 68 \\
M=1 / 19(5.3 \%)\end{array}$ & $3 / 5$ \\
\hline $\begin{array}{l}\text { Pillay \& Wassenaar } \\
\text { (1991) [87] } \\
\text { South Africa }\end{array}$ & $\begin{array}{l}\text { General } \\
\text { hospital }\end{array}$ & $\begin{array}{l}N=40 \\
(F=26, M=14)\end{array}$ & $\begin{array}{l}\text { Ingestion of: } \\
\text { - Medicines = 38/40 (95\%) } \\
\text { - Pesticides = 2/40 (5\%) }\end{array}$ & - & $3 / 5$ \\
\hline $\begin{array}{l}\text { Mhlongo \& Peltzer } \\
\text { (1999) [88] } \\
\text { South Africa }\end{array}$ & $\begin{array}{l}\text { General } \\
\text { hospital }\end{array}$ & $\begin{array}{l}N=100 \\
(F=63, M=37)\end{array}$ & $\begin{array}{l}\text { - Paraffin = 36/100 (36\%) } \\
\text { - Methylated spirit = } 12 / 100(12 \%) \\
\text { - Shampoo }=11 / 100(11 \%) \\
\text { - Pesticides }=10 / 100(10 \%) \\
\text { - Detergent = 9/100 (9\%) } \\
\text { - Battery acid =6/100 (6\%) } \\
\text { - Medicaments = 3/100 (3\%) } \\
\text { - Ingestion of glass = 4/100 (4\%) }\end{array}$ & Hanging = 9/100 (9\%) & $3 / 5$ \\
\hline $\begin{array}{l}\text { Madu \& Matla } \\
\text { (2003) [37] } \\
\text { South Africa }\end{array}$ & School & $\begin{array}{l}N=435 \\
(F=243, M=192)\end{array}$ & $\begin{array}{l}\text { - Self-poisoning }=40 / 435(9.2 \%) \\
F=21 / 243(8.6 \%) \\
M=19 / 192(9.9 \%) \\
\text { - Drug overdose }=23 / 435(5.3 \%) \\
F=13 / 243(5.3 \%) \\
M=10 / 192(5.2)\end{array}$ & $\begin{array}{l}\text { - Hanging }=20 / 435(4.6 \%) \\
F=5 / 243(2.1 \%) \\
M=15 / 192(7.8 \%) \\
\text { - Stabbing }=2 / 435(0.5 \%) \\
F=0 / 243 \\
M=2 / 192(1 \%)\end{array}$ & $4 / 5$ \\
\hline $\begin{array}{l}\text { Sommer } \\
\text { (2005) [39] } \\
\text { South Africa }\end{array}$ & School & $\begin{array}{l}N=299 \\
(F=185, M=114)\end{array}$ & - Overdose = 141/299 (47.2\%) & $\begin{array}{l}\text { - Wrist cutting = } 133 / 299(44.4 \%) \\
\text { - Jumping from height = 25/299 (8.4\%) }\end{array}$ & $2 / 5$ \\
\hline $\begin{array}{l}\text { Yéo-Tenena et al. (2010) } \\
\text { [89] } \\
\text { Ivory Coast }\end{array}$ & Hospital & $\begin{array}{l}N=42 \\
(F=33, M=9)\end{array}$ & $\begin{array}{l}\text { - } \text { Chloroquine = 26/42 (61.9\%) } \\
\text { - } \text { Psychotropic = } 8 / 42(19 \%) \\
\text { - } \text { Paracetamol = } 4 / 42(9.5 \%) \\
\text { - } \text { Metronidazole = } 3 / 42(7.1 \%) \\
\text { - } \text { Muriatic acid = 3/42 (7.1\%) } \\
\text { - } \text { Sodium hypochlorite = } 2 / 42(4.8 \%) \\
\text { - Ethyl alcohol = } 2 / 42(4.8 \%) \\
\text { - } \text { Rat poison = 2/42 (4.8\%) } \\
\text { - } \text { Thinner = } 1 / 42(2.4 \%)\end{array}$ & - Hanging = 1/42 (2.4\%) & $4 / 5$ \\
\hline $\begin{array}{l}\text { Beekrum et al. (2011) } \\
\text { [24] } \\
\text { South Africa }\end{array}$ & Hospital & $\begin{array}{l}N=10 \\
(F=10)\end{array}$ & $\begin{array}{l}\text { - All participants took overdose of } \\
\text { prescription medication belonging } \\
\text { to a family member: benzodiazepines, } \\
\text { steroidal anti-inflammatories, and } \\
\text { various blood pressure medications. }\end{array}$ & & $5 / 5$ \\
\hline $\begin{array}{l}\text { Okoko et al. } \\
\text { (2011) [90] } \\
\text { Congo Brazzaville }\end{array}$ & Hospital & $\begin{array}{l}N=62 \\
(F=50, M=12)\end{array}$ & $\begin{array}{l}\text { - Overdose = } 53(85.5 \%) \\
\text { - Caustic soda }=6(9.7 \%) \\
\text { - Powder }=1(1.6 \%) \\
\text { - Rat poison = } 1(1.6 \%)\end{array}$ & - Hanging = $1(1.6 \%)$ & $4 / 5$ \\
\hline Pretorius & Children's & $N=12$ & - & - Cutting = 11 (91.6\%) & $3 / 5$ \\
\hline
\end{tabular}


Table 3 Predominant Form / Method of Self-harm by year of publication (Continued)

\begin{tabular}{|c|c|c|c|c|c|}
\hline \multirow{2}{*}{$\begin{array}{l}\text { Author } \\
\text { (year) } \\
\text { Country }\end{array}$} & \multirow[t]{2}{*}{ Setting } & \multirow{2}{*}{$\begin{array}{l}\text { Sample } \\
\text { (Sex) }\end{array}$} & \multicolumn{2}{|l|}{ Reported method of self-harm } & \multirow{2}{*}{$\begin{array}{l}\text { Study } \\
\text { quality }\end{array}$} \\
\hline & & & Self-Poisoning & Self-Injury & \\
\hline $\begin{array}{l}(2011)[29] \\
\text { South Africa }\end{array}$ & homes & $(F=10, M=2)$ & & 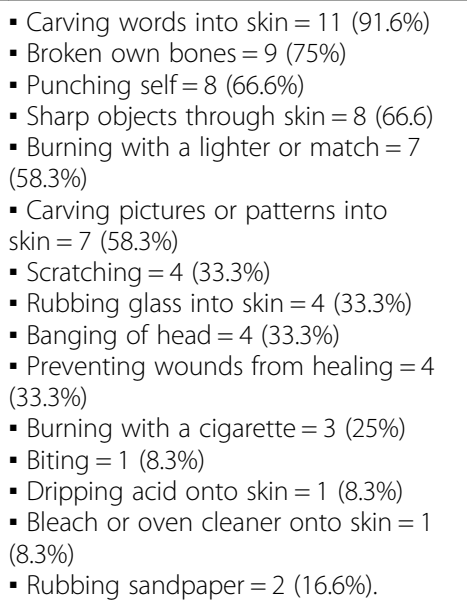 & \\
\hline $\begin{array}{l}\text { Fine et al. } \\
\text { (2012) [91] } \\
\text { South Africa }\end{array}$ & Hospital & $\begin{array}{l}N=50 \\
(F=31 \\
M=19)\end{array}$ & $\begin{array}{l}\text { - } \text { Overdose }=17 / 50(34 \%) \\
\text { - Poisoning }=2 / 50(4 \%) \\
\text { - Drowning }=2 / 50(4 \%)\end{array}$ & $\begin{array}{l}\text { - } \text { Cutting = } 36 / 50(75 \%) \\
\text { - Hanging }=10(20 \%) \\
\text { - Jumping from a height = 3/50 (6\%) }\end{array}$ & $3 / 5$ \\
\hline $\begin{array}{l}\text { Shilubane et al. (2012) } \\
\text { [32] } \\
\text { South Africa }\end{array}$ & Community & $\begin{array}{l}N=14 \\
(F=8, M=6)\end{array}$ & $\begin{array}{l}\text { Ingestion of: } \\
\text { - Medications = 9/14 (64.3\%) } \\
\text { - Paraffin =1/14 (7.1\%) } \\
\text { - Disinfectant = 1/14 (7.1) }\end{array}$ & $\begin{array}{l}\text { - } \text { Burning }=1 / 14(7.1 \%) \\
\text { - Hanging =1/14 (7.1\%) }\end{array}$ & $4 / 5$ \\
\hline $\begin{array}{l}\text { Van Rooyen } \\
(2013)^{\mathrm{a}}[50] \\
\text { Lippi } \\
(2014)^{\mathrm{a}}[52] \\
\text { South Africa }\end{array}$ & University & $\begin{array}{l}N=603 \\
(F=483, M=120)\end{array}$ & - & 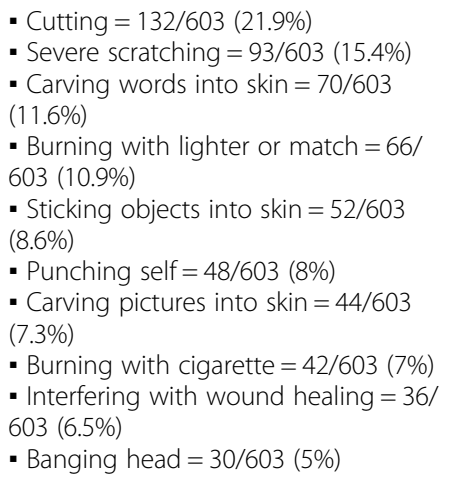 & $3 / 5$ \\
\hline $\begin{array}{l}\text { van der Walt } \\
\text { (2016) [60] } \\
\text { South Africa }\end{array}$ & University & $\begin{array}{l}N=201 \\
(F=110, M=91)\end{array}$ & $\begin{array}{l}\text { - Alcohol abuse = 46/201 (22.9\%) } \\
\text { - Overdose = 25/201 (12.4\%) } \\
\text { - Medication abuse =12/201 (6\%) }\end{array}$ & $\begin{array}{l}\text { - Hitting self }=26 / 201(12.9 \%) \\
\text { - Head banging =24/201 (11.9\%) } \\
\text { - } \text { Cutting=18/201 (9\%) } \\
\text { - Scratching = } 17 / 201(8.5 \%) \\
\text { - } \text { Exercised an injury on purpose = 12/ } \\
201(6 \%) \\
\text { - Prevented wounds from healing = 10/ } \\
201(5 \%) \\
\text { - } \text { Burning = } 4 / 201(2 \%) \\
\text { - } \text { Reckless driving }=21 / 201(10.4 \%)\end{array}$ & $3 / 5$ \\
\hline $\begin{array}{l}\text { Meissner \& Bantjes } \\
\text { (2017) [27] } \\
\text { South Africa }\end{array}$ & University & $N=4(M=4)$ & - & $\begin{array}{l}\text { - } \text { Hanging }=2 / 4(50 \%) \\
\text { - } \text { Car accident = 2/4 (50\%) }\end{array}$ & $5 / 5$ \\
\hline $\begin{array}{l}\text { Kritzinger } \\
\text { (2018) [26] } \\
\text { South Africa }\end{array}$ & Hospital & $N=10(F=10)$ & $\begin{array}{l}\text { - Overdose } \\
\text { - Rat poison } \\
\text { - Furniture oil }\end{array}$ & - & $5 / 5$ \\
\hline
\end{tabular}

\section{F Female}

${ }^{a}$ The studies by Lippi (2014) [52] and van Rooyen (2013) [50] were based on the same dataset, the 2009 University of Pretoria student survey in South Africa 
Table 4 Associates, Risk and Protective Factors of Self-Harm (by year of publication)

\begin{tabular}{|c|c|c|c|c|c|c|}
\hline \multirow{2}{*}{$\begin{array}{l}\text { Author } \\
\text { (year) } \\
\text { Country }\end{array}$} & \multicolumn{5}{|l|}{ Associates / Risk Factors } & \multirow{2}{*}{$\begin{array}{l}\text { Study } \\
\text { quality }\end{array}$} \\
\hline & Personal $^{\text {a }}$ & Family ${ }^{b}$ & School $^{\mathrm{c}}$ & Interpersonal $^{d}$ & Abuse and violence ${ }^{e}$ & \\
\hline $\begin{array}{l}\text { Cummins \& } \\
\text { Allwood } \\
\text { (1984) [83] } \\
\text { South Africa }\end{array}$ & - Psychiatric disturbance & $\begin{array}{l}\text { - Family dysfunction } \\
\text { (including divorce) } \\
\text { - Family psychiatric illness }\end{array}$ & $\begin{array}{l}\text { - School } \\
\text { problems }\end{array}$ & $\begin{array}{l}\text { - Socialisation } \\
\text { problems }\end{array}$ & - & $3 / 5$ \\
\hline $\begin{array}{l}\text { Pillay } \\
\text { (1987) [85] } \\
\text { South Africa }\end{array}$ & - Medical/psychiatric illness & $\begin{array}{l}\text { - Problems with Parents } \\
\text { - Problems with siblings } \\
\text { - Marital problems }\end{array}$ & $\begin{array}{l}\text { - School } \\
\text { problems }\end{array}$ & $\begin{array}{l}\text { - Problems with } \\
\text { boyfriend or girlfriend }\end{array}$ & - & $3 / 5$ \\
\hline $\begin{array}{l}\text { Sefa-Dedeh \& } \\
\text { Canetto } \\
\text { (1992) [22] } \\
\text { Ghana }\end{array}$ & - & $\begin{array}{l}\text { - Family harassment and } \\
\text { dispute } \\
\text { - Failed sense of autonomy } \\
\text { in the family }\end{array}$ & - & - & - & $4 / 5$ \\
\hline $\begin{array}{l}\text { Kebede \& } \\
\text { Ketsela } \\
\text { (1993) [35] } \\
\text { Ethiopia }\end{array}$ & $\begin{array}{l}\text { - Hopelessness } \\
\text { - Heavy alcohol intake }\end{array}$ & - & $\begin{array}{l}\text { - Lower } \\
\text { school } \\
\text { grade }\end{array}$ & - & - & $5 / 5$ \\
\hline $\begin{array}{l}\text { Pillay \& } \\
\text { Wassenaar } \\
\text { (1997) [95] } \\
\text { South Africa }\end{array}$ & - Depression & $\begin{array}{l}\text { - Lower family adaptability } \\
\text { - Low family cohesion } \\
\text { - Low family satisfaction } \\
\text { - Hopelessness } \\
\text { - Psychiatric disturbances. }\end{array}$ & $\begin{array}{l}\text { - Problems } \\
\text { at school }\end{array}$ & $\begin{array}{l}\text { - Romantic } \\
\text { relationship problems }\end{array}$ & - & $5 / 5$ \\
\hline $\begin{array}{l}\text { Wassenaar } \\
\text { et al. } \\
\text { (1998) [23] } \\
\text { South Africa }\end{array}$ & - Hopelessness & $\begin{array}{l}\text { - Family communication } \\
\text { breakdown } \\
\text { - Conflict with parents } \\
\text { - Authoritarian patriarchy. }\end{array}$ & - & - & - & $4 / 5$ \\
\hline $\begin{array}{l}\text { Mhlongo \& } \\
\text { Peltzer } \\
\text { (1999) [88] } \\
\text { South Africa }\end{array}$ & $\begin{array}{l}\text { - AIDS phobia } \\
\text { - Teenage pregnancy } \\
\text { - Mental illness } \\
\text { - Unemployment } \\
\text { - Financial problems }\end{array}$ & - Problem with parents & $\begin{array}{l}\text { - Academic } \\
\text { failure }\end{array}$ & $\begin{array}{l}\text { - Romantic } \\
\text { relationship problems }\end{array}$ & - & $3 / 5$ \\
\hline $\begin{array}{l}\text { Peltzer et al. } \\
\text { (2000) [36] } \\
\text { South Africa }\end{array}$ & $\begin{array}{l}\text { - Suicidal ideation } \\
\text { - Suicide intent }\end{array}$ & $\begin{array}{l}\text { - History of family suicide } \\
\text { - Parental divorced } \\
\text { - Large family size }\end{array}$ & - & $\begin{array}{l}\text { - History of suicide by } \\
\text { friend }\end{array}$ & - & $3 / 5$ \\
\hline $\begin{array}{l}\text { Madu \& Matla } \\
\text { (2004) [96] } \\
\text { South Africa }\end{array}$ & - & - Family conflict & - & - & - & $4 / 5$ \\
\hline $\begin{array}{l}\text { Wild et al. } \\
\text { (2004) [38] } \\
\text { South Africa }\end{array}$ & $\begin{array}{l}\text { - Depression } \\
\text { - Poor global self-worth } \\
\text { - Poor body image } \\
\text { - Female sex }\end{array}$ & - & $\begin{array}{l}\text { - Poor } \\
\text { schoolwork }\end{array}$ & - Problems with peers & - & $4 / 5$ \\
\hline $\begin{array}{l}\text { Sommer } \\
\text { (2005) [39] } \\
\text { South Africa }\end{array}$ & $\begin{array}{l}\text { - Female sex } \\
\text { - Previous psychiatric contact }\end{array}$ & $\begin{array}{l}\text { - Perceived lack of family } \\
\text { support } \\
\text { - Suicide attempt in the } \\
\text { family }\end{array}$ & - & - Death of a friend & - & $2 / 5$ \\
\hline $\begin{array}{l}\text { Shiferaw et al. } \\
\text { (2006) [41] } \\
\text { Ethiopia }\end{array}$ & $\begin{array}{l}\text { - Being sexually active } \\
\text { - Female sex } \\
\text { - Unwanted pregnancy } \\
\text { - Boredom } \\
\text { - HIV/AIDS positive status }\end{array}$ & $\begin{array}{l}\text { - Family member } \\
\text { attempted suicide } \\
\text { - Lack of family support } \\
\text { - Living with both } \\
\text { biological parents }\end{array}$ & $\begin{array}{l}\text { - Academic } \\
\text { under- } \\
\text { achievement }\end{array}$ & $\begin{array}{l}\text { - Friend suicide } \\
\text { attempt } \\
\text { - Romantic } \\
\text { relationship problems }\end{array}$ & - & $2 / 5$ \\
\hline $\begin{array}{l}\text { Omigbodun } \\
\text { et al. } \\
\text { (2008) [20] } \\
\text { Nigeria }\end{array}$ & $\begin{array}{l}\text { - Drinking alcohol } \\
\text { - Having to go hungry }\end{array}$ & $\begin{array}{l}\text { - Unstable family life } \\
\text { - Having a mother who } \\
\text { had been married more } \\
\text { than once } \\
\text { - Living in urban location }\end{array}$ & - & - & $\begin{array}{l}\text { - Sexual abuse } \\
\text { - Physical attack } \\
\text { - Physical fights }\end{array}$ & $4 / 5$ \\
\hline $\begin{array}{l}\text { Yéo-Tenena } \\
\text { et al. } \\
\text { (2010) [89] } \\
\text { Ivory Coast }\end{array}$ & $\begin{array}{l}\text { - Psychiatric problems } \\
\text { (depression, substance } \\
\text { addiction) } \\
\text { - Previous suicide attempt } \\
\text { - Emotional problems }\end{array}$ & - Familial conflict & $\begin{array}{l}\text { - School } \\
\text { failure }\end{array}$ & $\begin{array}{l}\text { - Unwanted } \\
\text { pregnancy }\end{array}$ & - Sexual abuse & $4 / 5$ \\
\hline
\end{tabular}


Table 4 Associates, Risk and Protective Factors of Self-Harm (by year of publication) (Continued)

\begin{tabular}{|c|c|c|c|c|c|c|}
\hline \multirow{2}{*}{$\begin{array}{l}\text { Author } \\
\text { (year) } \\
\text { Country }\end{array}$} & \multicolumn{5}{|l|}{ Associates / Risk Factors } & \multirow{2}{*}{$\begin{array}{l}\text { Study } \\
\text { quality }\end{array}$} \\
\hline & Personal ${ }^{a}$ & Family $^{b}$ & School $^{c}$ & Interpersonal ${ }^{d}$ & Abuse and violence ${ }^{e}$ & \\
\hline $\begin{array}{l}\text { Beekrum et al. } \\
\text { (2011) [24] } \\
\text { South Africa }\end{array}$ & - Hopelessness and despair & $\begin{array}{l}\text { - Previous suicide or } \\
\text { attempted suicide by a } \\
\text { close family member } \\
\text { - Conflictual, disengaged } \\
\text { or over-protective family } \\
\text { relationships } \\
\text { - Strained adolescent- } \\
\text { parent communication } \\
\text { - Conflicting social roles } \\
\text { and values in the context } \\
\text { of contemporary } \\
\text { acculturation pressures }\end{array}$ & $\begin{array}{l}\text { - Academic } \\
\text { failure }\end{array}$ & $\begin{array}{l}\text { - Breakup } \\
\text { - Lack of social } \\
\text { support }\end{array}$ & $\begin{array}{l}\text { - Physical and } \\
\text { emotional abuse in the } \\
\text { family }\end{array}$ & $5 / 5$ \\
\hline $\begin{array}{l}\text { Okoko et al. } \\
\text { (2011) [90] } \\
\text { The Congo }\end{array}$ & $\begin{array}{l}\text { - Previous suicide attempt } \\
\text { - Psychosis } \\
\text { - Alcohol abuse } \\
\text { - Drugs abuse } \\
\text { - Emotional breakdown }\end{array}$ & $\begin{array}{l}\text { - Conflict with parents } \\
\text { - Difficulty with family } \\
\text { communication } \\
\text { - Parental divorce } \\
\text { - Parental death } \\
\text { - Kinship fostering } \\
\text { - Living in a stepfamily }\end{array}$ & $\begin{array}{l}\text { - School } \\
\text { problems }\end{array}$ & - Breakup & $\begin{array}{l}\text { - Domestic violence } \\
\text { victimisation } \\
\text { - Sexual abuse } \\
\text { - Neglect } \\
\text { - Incest }\end{array}$ & $4 / 5$ \\
\hline $\begin{array}{l}\text { Pretorius } \\
\text { (2011) [29] } \\
\text { South Africa }\end{array}$ & $\begin{array}{l}\text { - Personal history of suicide } \\
\text { attempts suicide; } \\
\text { - Previous diagnosis of mood } \\
\text { disorders (i.e., major } \\
\text { depression, and bipolar } \\
\text { disorder) }\end{array}$ & $\begin{array}{l}\text { - Experience of human } \\
\text { trafficking before removal } \\
\text { from parental care } \\
\text { - Dysfunctional parenting } \\
\text { (unavailability, conflict, or } \\
\text { alcoholism) before removal } \\
\text { from parental care } \\
\text { - Family history of } \\
\text { attempted suicide }\end{array}$ & - & $\begin{array}{l}\text { - Observation of the } \\
\text { self-harm of another } \\
\text { adolescent at the } \\
\text { same children's home }\end{array}$ & $\begin{array}{l}\text { - Abuse (i.e., physical, } \\
\text { sexual, and emotional } \\
\text { abuse) before removal } \\
\text { from parental care. }\end{array}$ & $3 / 5$ \\
\hline $\begin{array}{l}\text { Campbell } \\
\text { (2012) [45] } \\
\text { South Africa }\end{array}$ & $\begin{array}{l}\text { - Female sex; } \\
\text { - Coloured race; }\end{array}$ & $\begin{array}{l}\text { - Stressful relationships } \\
\text { with parents and extended } \\
\text { family } \\
\text { - Financial hardship }\end{array}$ & - & $\begin{array}{l}\text { - Stressful romantic } \\
\text { relationship } \\
\text { - Negative life events }\end{array}$ & - & $3 / 5$ \\
\hline $\begin{array}{l}\text { Shilubane } \\
\text { et al. } \\
\text { (2012) [32] } \\
\text { South Africa }\end{array}$ & $\begin{array}{l}\text { - Perceived accusations of } \\
\text { negative behaviour } \\
\text { - Feelings of physical } \\
\text { rejection } \\
\text { - Acute negative mood (e.g., } \\
\text { depression, anger, } \\
\text { hopelessness) } \\
\text { - Being unaware of } \\
\text { community-support } \\
\text { resources } \\
\text { - Personal history of } \\
\text { attempted suicide }\end{array}$ & $\begin{array}{l}\text { - Conflictual and strained } \\
\text { family relationships } \\
\text { - Lack of family support } \\
\text { - Family member HIV } \\
\text { positive status } \\
\text { - Death of close family } \\
\text { member } \\
\text { - Family history of } \\
\text { attempted suicide } \\
\text { - Family poverty }\end{array}$ & - & $\begin{array}{l}\text { - Lack of trusted peer } \\
\text { support } \\
\text { - Peer suicide attempt }\end{array}$ & - & $4 / 5$ \\
\hline $\begin{array}{l}\text { Swahn et al. } \\
\text { (2012) [30] } \\
\text { Uganda }\end{array}$ & $\begin{array}{l}\text { - Sadness } \\
\text { - Expectations of dying prior } \\
\text { to age } 30\end{array}$ & $\begin{array}{l}\text { - Parental neglect due to } \\
\text { alcohol use }\end{array}$ & - & - & - & $4 / 5$ \\
\hline $\begin{array}{l}\text { Vawda } \\
\text { (2012) [47] } \\
\text { South Africa }\end{array}$ & - & - Family member suicide & - & - & - & $3 / 5$ \\
\hline $\begin{array}{l}\text { Gage } \\
\text { (2013) [25] } \\
\text { Ethiopia }\end{array}$ & $\begin{array}{l}\text { - Currently employed } \\
\text { - Lost much sleep over worry } \\
\text { - Depression }\end{array}$ & $\begin{array}{l}\text { - Receiving marriage } \\
\text { request } \\
\text { - Both parents deceased }\end{array}$ & - & $\begin{array}{l}\text { - Community } \\
\text { involvement in child } \\
\text { marriage prevention }\end{array}$ & $\begin{array}{l}\text { - Sexual violence } \\
\text { victimisation }\end{array}$ & $4 / 5$ \\
\hline $\begin{array}{l}\text { Muula et al. } \\
\text { (2013) [48] } \\
\text { Zambia }\end{array}$ & $\begin{array}{l}\text { - Female sex } \\
\text { - Aged } \leq 14 \text { yrs. } \\
\text { - Loneliness } \\
\text { - Sleeplessness due to worry } \\
\text { - Hopelessness } \\
\text { - Suicidal ideation } \\
\text { - Marijuana use } \\
\text { - Drunkenness }\end{array}$ & - & - & - & - Use of dagga & $5 / 5$ \\
\hline
\end{tabular}


Table 4 Associates, Risk and Protective Factors of Self-Harm (by year of publication) (Continued)

\begin{tabular}{|c|c|c|c|c|c|c|}
\hline \multirow{2}{*}{$\begin{array}{l}\text { Author } \\
\text { (year) } \\
\text { Country }\end{array}$} & \multicolumn{5}{|l|}{ Associates / Risk Factors } & \multirow{2}{*}{$\begin{array}{l}\text { Study } \\
\text { quality }\end{array}$} \\
\hline & Personal $^{\text {a }}$ & Family ${ }^{b}$ & School $^{c}$ & Interpersonal $^{d}$ & Abuse and violence ${ }^{e}$ & \\
\hline $\begin{array}{l}\text { Shilubane } \\
\text { et al. } \\
\text { (2013) [49] } \\
\text { South Africa }\end{array}$ & $\begin{array}{l}\text { - Female sex } \\
\text { - Hopelessness } \\
\text { - Feeling unsafe } \\
\text { - Substance use } \\
\text { - Having unsafe sex } \\
\text { - Older adolescence } \\
\text { - Body dissatisfaction. }\end{array}$ & - & $\begin{array}{l}\text { - Lower } \\
\text { grade }\end{array}$ & - & - Violence & $5 / 5$ \\
\hline $\begin{array}{l}\text { Chinawa et al. } \\
\text { (2014) [51] } \\
\text { Nigeria }\end{array}$ & $\begin{array}{l}\text { - Depression } \\
\text { - Alcohol and drug use }\end{array}$ & - & - & - & - & $2 / 5$ \\
\hline $\begin{array}{l}\text { Penning \& } \\
\text { Collings } \\
\text { (2014) [53] } \\
\text { South Africa }\end{array}$ & - Female sex & - Domestic injury & - & - & $\begin{array}{l}\text { - Domestic assault } \\
\text { - Rape } \\
\text { - Emotional abuse } \\
\text { - Negative child sexual } \\
\text { abuse appraisals }\end{array}$ & $5 / 5$ \\
\hline $\begin{array}{l}\text { Randall et al. } \\
\text { (2014) [54] } \\
\text { Benin }\end{array}$ & $\begin{array}{l}\text { - Male sex } \\
\text { - Anxiety } \\
\text { - Loneliness } \\
\text { - Substance use }\end{array}$ & - & - & - & - Being attacked & $5 / 5$ \\
\hline $\begin{array}{l}\text { Lippi } \\
\text { (2014) [52] } \\
\text { South Africa }\end{array}$ & - Severe depression & - & - & - & & $3 / 5$ \\
\hline $\begin{array}{l}\text { Cluver et al. } \\
\text { (2015) [56] } \\
\text { South Africa }\end{array}$ & $\begin{array}{l}\text { - Older adolescence } \\
\text { - Female sex } \\
\text { - Orphanhood by AIDS, } \\
\text { - Previous suicide attempt }\end{array}$ & $\begin{array}{l}\text { - Parental AIDS-illness } \\
\text { - Food insecurity }\end{array}$ & - & - & $\begin{array}{l}\text { - Severe physical abuse } \\
\text { - Severe emotional } \\
\text { abuse } \\
\text { - Sexual abuse or rape } \\
\text { - Community violence } \\
\text { - Domestic violence } \\
\text { - Orphanhood by } \\
\text { homicide }\end{array}$ & $4 / 5$ \\
\hline $\begin{array}{l}\text { Ng et al. } \\
\text { (2015) [57] } \\
\text { Rwanda }\end{array}$ & $\begin{array}{l}\text { - Child mental health } \\
\text { symptoms (i.e., Depression } \\
\text { above diagnostic threshold; } \\
\text { conduct problems). }\end{array}$ & - Parenting style & - & - & - & $5 / 5$ \\
\hline $\begin{array}{l}\text { Giru } \\
(2016)[58] \\
\text { Ethiopia }\end{array}$ & $\begin{array}{l}\text { - Family history of suicide } \\
\text { - Loneliness } \\
\text { - Hopelessness } \\
\text { - Mental illness } \\
\text { - Financial loss }\end{array}$ & $\begin{array}{l}\text { - Family conflict } \\
\text { - Death in family }\end{array}$ & $\begin{array}{l}\text { - Academic } \\
\text { failure }\end{array}$ & $\begin{array}{l}\text { - Lack of social } \\
\text { support }\end{array}$ & - & $3 / 5$ \\
\hline $\begin{array}{l}\text { Shaikh et al. } \\
\text { (2016) [59] } \\
\text { Malawi }\end{array}$ & $\begin{array}{l}\text { - Female sex } \\
\text { - Early sexual debut } \\
\text { - Serious injury } \\
\text { - Loneliness } \\
\text { - Anxiety } \\
\text { - Suicide ideation } \\
\text { - Suicide planning } \\
\text { - Alcohol use }\end{array}$ & - Parental tobacco use & - & $\begin{array}{l}\text { - Lifetime sexual } \\
\text { partners } \\
\text { - Number of days } \\
\text { people smoked in } \\
\text { presence weekly } \\
\text { - Having many close } \\
\text { friends }\end{array}$ & $\begin{array}{l}\text { - Bullied } \\
\text { - Physical fight } \\
\text { - Physically attacked } \\
\text { - Physically bullied }\end{array}$ & $5 / 5$ \\
\hline $\begin{array}{l}\text { Asante et al. } \\
\text { (2017) [62] } \\
\text { Ghana }\end{array}$ & $\begin{array}{l}\text { - Anxiety } \\
\text { - Loneliness }\end{array}$ & - Parental understanding & - & $\begin{array}{l}\text { - Food insecurity } \\
\text { - Having many close } \\
\text { friends }\end{array}$ & $\begin{array}{l}\text { - Bullied } \\
\text { - Being attacked } \\
\text { - Fighting }\end{array}$ & $5 / 5$ \\
\hline $\begin{array}{l}\text { Asante \& } \\
\text { Meyer-Weitz } \\
\text { (2017) [31] } \\
\text { Ghana }\end{array}$ & $\begin{array}{l}\text { - Female sex } \\
\text { - Aged } 15 \text { years or older } \\
\text { - Smoking } \\
\text { - Past alcohol use } \\
\text { - Present alcohol use } \\
\text { - Marijuana use } \\
\text { - Survival sex }\end{array}$ & - & - & - & $\begin{array}{l}\text { - Assaulted with a } \\
\text { weapon } \\
\text { - Having been robbed }\end{array}$ & $4 / 5$ \\
\hline $\begin{array}{l}\text { Peltzer \& } \\
\text { Pengpid } \\
\text { (2017) [65] }\end{array}$ & $\begin{array}{l}\text { - Health risk behaviours } \\
\text { - Hunger } \\
\text { - Parental support }\end{array}$ & - & - & - & - & $4 / 5$ \\
\hline
\end{tabular}


Table 4 Associates, Risk and Protective Factors of Self-Harm (by year of publication) (Continued)

\begin{tabular}{|c|c|c|c|c|c|c|}
\hline \multirow{2}{*}{$\begin{array}{l}\text { Author } \\
\text { (year) } \\
\text { Country }\end{array}$} & \multicolumn{5}{|l|}{ Associates / Risk Factors } & \multirow{2}{*}{$\begin{array}{l}\text { Study } \\
\text { quality }\end{array}$} \\
\hline & Personal $^{a}$ & Family $^{b}$ & School $^{\mathrm{c}}$ & Interpersonal $^{d}$ & Abuse and violence ${ }^{\mathrm{e}}$ & \\
\hline \multicolumn{7}{|l|}{ Namibia } \\
\hline $\begin{array}{l}\text { Amare et al. } \\
\text { (2018) [67] } \\
\text { Ethiopia }\end{array}$ & $\begin{array}{l}\text { - Living alone } \\
\text { - Loneliness } \\
\text { - Hopelessness } \\
\text { - Sleep disturbance worries } \\
\text { - Being physically hurt }\end{array}$ & - & - Truancy & - Poor social support & - & $5 / 5$ \\
\hline $\begin{array}{l}\text { Khuzwayo } \\
\text { et al. } \\
\text { (2018) [68] } \\
\text { South Africa }\end{array}$ & $\begin{array}{l}\text { - Aged } 16 \text { years and above } \\
\text { - Female sex } \\
\text { - Cannabis use }\end{array}$ & - & - & - & $\begin{array}{l}\text { - Threatened in school } \\
\text { with a weapon } \\
\text { - Bullied in school } \\
\text { - Dating violence } \\
\text { victimisation } \\
\text { - Cyber bullying }\end{array}$ & $3 / 5$ \\
\hline $\begin{array}{l}\text { Kritzinger } \\
\text { (2018) [26] } \\
\text { South Africa }\end{array}$ & $\begin{array}{l}\text { - Anger } \\
\text { - Low mood } \\
\text { - Suicidal ideation } \\
\text { - Previous suicide attempt } \\
\text { - Impulsivity } \\
\text { - Unemployment }\end{array}$ & - Conflict with parents & - & $\begin{array}{l}\text { - Breakup } \\
\text { - Loss of significant } \\
\text { other }\end{array}$ & $\begin{array}{l}\text { - Domestic abuse } \\
\text { victimisation }\end{array}$ & $5 / 5$ \\
\hline $\begin{array}{l}\text { van der Wal \& } \\
\text { George } \\
\text { (2018) [70] } \\
\text { South Africa }\end{array}$ & $\begin{array}{l}\text { - Emotional reactivity } \\
\text { - Tension-reduction coping }\end{array}$ & - & - & - Social support & - & $3 / 5$ \\
\hline $\begin{array}{l}\text { Baiden et al. } \\
\text { (2019) [71] } \\
\text { Ghana }\end{array}$ & $\begin{array}{l}\text { - Anxiety } \\
\text { - Illicit substance use } \\
\text { - Physical activity }\end{array}$ & - & - & - Having a close friend & - Bullying victimisation & $5 / 5$ \\
\hline $\begin{array}{l}\text { Carvalho et al. } \\
\text { (2019) [97] } \\
\text { Cross-national } \\
\text { study (Benin, } \\
\text { Ghana, } \\
\text { Mozambique, } \\
\text { Namibia, \& } \\
\text { Tanzania). }\end{array}$ & - Cannabis use & - & - & - & - & $5 / 5$ \\
\hline $\begin{array}{l}\text { Darré et al. } \\
\text { (2019) [73] } \\
\text { Togo }\end{array}$ & $\begin{array}{l}\text { - Female sex } \\
\text { - Being aged > } 18 \\
\text { - Sentimental problems } \\
\text { - Health problems } \\
\text { - Loneliness } \\
\text { - Unwanted pregnancy } \\
\text { - Distaste of life } \\
\text { - Abstinence }\end{array}$ & $\begin{array}{l}\text { - Family history of suicide } \\
\text { - Financial problems } \\
\text { - Family problems } \\
\text { - Absence of parents }\end{array}$ & - & $\begin{array}{l}\text { - Living as a couple } \\
\text { - Death of a loved one }\end{array}$ & - & $3 / 5$ \\
\hline $\begin{array}{l}\text { Koyanagi, Oh } \\
\text { et al. } \\
\text { (2019) [74] } \\
\text { Cross-national } \\
\text { study } \\
\text { (Benin, Ghana, } \\
\text { Malawi, } \\
\text { Mauritania, } \\
\text { Mozambique, } \\
\text { Namibia, } \\
\text { Seychelles, } \\
\text { Swaziland, \& } \\
\text { Tanzania). }\end{array}$ & - & - & - & - & - Bullying victimisation & $5 / 5$ \\
\hline $\begin{array}{l}\text { Koyanagi, } \\
\text { Stubbs, et al. } \\
\text { (2019) [75] } \\
\text { Cross-national } \\
\text { (Benin, Ghana, } \\
\text { Mauritania, } \\
\text { Mozambique, }\end{array}$ & - & $\begin{array}{l}\text { - Children and adolescent } \\
\text { food insecurity }\end{array}$ & - & - & - & $5 / 5$ \\
\hline
\end{tabular}


Table 4 Associates, Risk and Protective Factors of Self-Harm (by year of publication) (Continued)

\begin{tabular}{|c|c|c|c|c|c|c|}
\hline \multirow{2}{*}{$\begin{array}{l}\text { Author } \\
\text { (year) } \\
\text { Country }\end{array}$} & \multicolumn{5}{|l|}{ Associates / Risk Factors } & \multirow{2}{*}{$\begin{array}{l}\text { Study } \\
\text { quality }\end{array}$} \\
\hline & Personal $^{a}$ & Family ${ }^{b}$ & School $^{\mathrm{c}}$ & Interpersonal $^{d}$ & Abuse and violence ${ }^{e}$ & \\
\hline \multicolumn{7}{|l|}{$\begin{array}{l}\text { Namibia, } \\
\text { Seychelles, } \\
\text { Swaziland, \& } \\
\text { Tanzania). }\end{array}$} \\
\hline $\begin{array}{l}\text { Nguyen et al. } \\
\text { (2019) [76] } \\
\text { Cross-national } \\
\text { (Nigeria, } \\
\text { Uganda, \& } \\
\text { Zambia). }\end{array}$ & - & $\begin{array}{l}\text { - Orphanhood prior to age } \\
18\end{array}$ & - & - & $\begin{array}{l}\text { - Coerced/forced sexual } \\
\text { initiation }\end{array}$ & $5 / 5$ \\
\hline $\begin{array}{l}\text { Shayo \& } \\
\text { Lawala } \\
\text { (2019) [78] } \\
\text { Tanzania }\end{array}$ & $\begin{array}{l}\text { - Loneliness } \\
\text { - Anxiety } \\
\text { - Younger age }\end{array}$ & $\begin{array}{l}\text { - Food insecurity } \\
\text { - Parental care }\end{array}$ & - & - & - & $5 / 5$ \\
\hline $\begin{array}{l}\text { Thornton et al. } \\
\text { (2019) [79] } \\
\text { Cross-national } \\
\text { (South Africa \& } \\
\text { Guyana) }\end{array}$ & - & - & - & - Social stress & - & $2 / 5$ \\
\hline $\begin{array}{l}\text { Vancampfort } \\
\text { et al. } \\
\text { (2019) [82] } \\
\text { Cross-national } \\
\text { (Benin, Ghana, } \\
\text { Mauritania, } \\
\text { Mozambique, } \\
\text { Namibia, } \\
\text { Seychelles, \& } \\
\text { Tanzania) }\end{array}$ & - Sedentary leisure-time & - & - & - & - & $5 / 5$ \\
\hline
\end{tabular}

apersonal level factors: These include personal characteristics and histories, and factors related to personal (mental) health conditions

${ }^{b}$ Family level factors: These cover factors and circumstances within the family, and relationships and interactions with family members

'School-level factors: These relate to academic performance and relationships and circumstances within the school context

${ }^{\mathrm{d}}$ Interpersonal level factors: These are circumstances related to the individual's relationships with peers and neighbours, and other social relationships and

interactions outside the family and school contexts

eAbuse and violence: Based on previous evidence, we created this category to include all abuse and violence items - that is psychological, physical, emotional,

and sexual abuse victimisation items

sub-Saharan Africa due to poverty, unemployment, death of parents (to AIDS), physical and sexual abuses including (forced) child marriage - displacement by wars and conflicts, substance use and abuse (due to less than ideally regulated access to prescription medication), family conflict, among others $[99,100]$. Under the circumstances, it is surprising that rates are not higher in African studies.

Findings from the qualitative studies in this review suggest some reasons for the high levels of self-harm among young people: entrenched cultural and family rules of comportment and norms of obedience and respect and the sense of powerlessness experienced by both boys and girls as linked to self-harm [22-24, 27, 32]. The higher prevalence estimates in females have been attributed to socially and religiously sanctioned oppression and exploitative normative gender role discrimination against women and girls [101]. Compared to young males, young females tend to be victims of more domestic chore burdens, caretaking responsibilities, sexual abuse and exploitation, exclusion from education, unemployment, and exclusion from decision making $[11,101]$. Thus, as found in regional reviews of studies involving adult samples $[11,12]$ and psychological autopsy studies of suicide in Africa [102, 103], self-harm and suicide among women has been interpreted as protestation against socially sanctioned abuse and oppressive control, while men's selfharm and suicide represent a quest for lost masculinity.

\section{Strengths and limitations of this review}

Our comprehensive search strategy identified a substantial literature in a previously under-reviewed geographical area. Of the 74 studies, $12(16.2 \%)$ were peerreviewed articles exclusively indexed in the African regional academic databases searched. These 12 papers represent a valuable addition to previous reports covering parts of sub-Saharan Africa [10].

Our categorisation of the factors associated with selfharm into personal, family, school, interpersonal level factors was motivated by the wide variation and the 
Table 5 Reported Reasons for Self-harm (by year of publication)

\begin{tabular}{|c|c|c|c|c|c|}
\hline \multirow{2}{*}{$\begin{array}{l}\text { Author } \\
\text { (year) } \\
\text { Country }\end{array}$} & \multirow[t]{2}{*}{ Term } & \multirow{2}{*}{$\begin{array}{l}\text { Setting. } \\
\text { Design. } \\
\text { (Sample). }\end{array}$} & \multicolumn{2}{|l|}{ Reported Reasons (n [\%]) } & \multirow{2}{*}{$\begin{array}{l}\text { Study } \\
\text { qualit }\end{array}$} \\
\hline & & & Intrapersonal Reasons $^{a}$ & Interpersonal Reasons $^{b}$ & \\
\hline $\begin{array}{l}\text { Sefa-Dedeh } \\
\& \text { Canetto } \\
\text { (1992) [22] } \\
\text { Ghana }\end{array}$ & $\begin{array}{l}\text { Attempted } \\
\text { suicide }\end{array}$ & $\begin{array}{l}\text { General Hospital. } \\
\text { Qualitative clinical case study of clinical } \\
\text { records. } \\
\text { (Two cases: Only Case A included in } \\
\text { review). }\end{array}$ & $\begin{array}{l}\text { To: } \\
\text { - Die } \\
\text { - Validate self }\end{array}$ & $\begin{array}{l}\text { To: } \\
\text { - Get revenge against parents } \\
\text { - Make parents feel guilty; } \\
\text { - Obtain empathy and understanding from family. } \\
\text { - Regain control over social relationships and } \\
\text { resources. }\end{array}$ & $4 / 5$ \\
\hline Wassenaar & Attempted & General Hospital. & To die & To resolve conflict with parents. & $4 / 5$ \\
\hline
\end{tabular}

\begin{tabular}{|c|c|c|}
\hline $\begin{array}{l}\text { et al. } \\
\text { (1998) [23] } \\
\text { South } \\
\text { Africa }\end{array}$ & suicide & $\begin{array}{l}\text { Qualitative clinical case } \\
\text { records. } \\
\text { (Three cases: Only Case } \\
\text { review). }\end{array}$ \\
\hline $\begin{array}{l}\text { Mhlongo \& } \\
\text { Peltzer } \\
\text { (1999) [88] } \\
\text { South } \\
\text { Africa }\end{array}$ & Parasuicide & $\begin{array}{l}\text { General hospital. } \\
\text { Patients' records and int } \\
\text { patients presenting with } \\
(n=100)\end{array}$ \\
\hline $\begin{array}{l}\text { Beekrum } \\
\text { et al. } \\
\text { (2011) [24] } \\
\text { South }\end{array}$ & $\begin{array}{l}\text { Non-fatal } \\
\text { suicidal } \\
\text { behaviour }\end{array}$ & $\begin{array}{l}\text { General hospital. } \\
\text { Qualitative case study. } \\
(n=10)\end{array}$ \\
\hline
\end{tabular}

Africa

$\begin{array}{lll}\text { Pretorius } & \text { Deliberate } & \text { Children's homes. } \\ \text { (2011) [29] } & \text { self-harm } & \begin{array}{l}\text { Mixed methods approach. } \\ (n=12)\end{array} \\ \text { South } & & \\ \text { Africa } & & \end{array}$

$\begin{array}{lll}\text { van Rooyen } & \text { Deliberate } & \text { University. } \\ \text { (2013) [50] } & \text { self-harm } & \text { Cross-sectional survey of students. } \\ \text { South } & & (n=603)\end{array}$

\begin{tabular}{|c|c|c|}
\hline $\begin{array}{l}\text { Meissner \& } \\
\text { Bantjes } \\
\text { (2017) [27] } \\
\text { South } \\
\text { Africa }\end{array}$ & $\begin{array}{l}\text { Attempted } \\
\text { suicide }\end{array}$ & $\begin{array}{l}\text { University. } \\
\text { One-to-one semi-structured qualitative in- } \\
\text { terviews with students with histories of } \\
\text { attempted suicide. } \\
(n=4)\end{array}$ \\
\hline $\begin{array}{l}\text { Kritzinger } \\
\text { (2018) [26] } \\
\text { South } \\
\text { Africa }\end{array}$ & $\begin{array}{l}\text { Non-Fatal } \\
\text { Suicidal } \\
\text { Behaviour }\end{array}$ & $\begin{array}{l}\text { General hospital. } \\
\text { Qualitative case study approach: One-to- } \\
\text { one semi-structured interviews with clin- } \\
\text { ical sample of adolescents. } \\
(n=10)\end{array}$ \\
\hline
\end{tabular}

To:

- Stop bad feelings (8

[66.6\%])

- Feel relaxed (7 [58.3\%])

- Feel something, even if

it was pain (7 [58.3\%])

- Punish self (5 [41.6\%])

- Relieve feeling 'numb'/ empty (5 [41.6\%])

To: ${ }^{c}$

- Stop bad feeling

- Relieve feeling numb or

empty

- Punish yourself

- Feel relaxed

- Get control of a

situation

- Feel part of a group

- Be like someone you

respect

- Avoid having to do something unpleasant you don't want to do

To: ${ }^{c}$

- Escape feeling trapped

- Avoid suicide

- Distract from painful

memories

- Die

To: ${ }^{c}$

- Escape unbearable

thoughts

- End sense of

meaninglessness

- Die
To demonstrate, usually, against family conflicts and abuse (58 [58\%])

To: ${ }^{c}$

- Let others (e.g., boyfriend, or parent) change

their behaviour or attitudes.

- Communicate distress related to conflict with parents, parental conflict, high parental expectations, and peer-cultural conflict. - Get parents/family to understand their problems.

To:

- Get control of a situation (5 [41.6\%])

- Receive more attention from guardians

/caregivers/ friends (2 [16.6\%])

- Get guardians/caregivers to understand you (2

[16.6\%])

- Get help (1 [8.3\%])

To: ${ }^{c}$

- Let others know how desperate you were

- Try to get a reaction from someone, even if it's a negative reaction

- Receive more attention from your parents or friends

- Get your parents to understand or notice you

- Get other people to act differently or change

- Avoid school, work, or other activities

- Avoid being with people

(1)

\footnotetext{
a Intrapersonal reasons (i.e., reasons intended to change one's state or circumstances): reasons or motives relate to desired changes in one's personal or internal state, including changes in sensations, emotional states or thoughts

b Interpersonal reasons (i.e., reasons intended to change the state or circumstances of significant others): include desired changes within one's social environment, such as communicating distress to someone, or to influence the behaviour of others or to punish others

c Frequency distribution of reasons not reported
} 
general lack of meaningful classification of these factors across the studies and is not without limitations. For example, in terms of the factors associated with self-harm, there is likely to be significant inherent interdependence between, for example, personal level factors and family level factors - family factors could be influencing the onset of the personal level factors and vice-versa - or between personal, school and interpersonal levels. The categorisation into intrapersonal and interpersonal factors too is therefore likely to be subject to considerable interdependence.

\section{Future directions}

Future studies should explore the prevalence estimates of self-harm among young people in non-clinical contexts such as the community and schools [6-8]. Participants in such prevalence studies should include other minority and vulnerable groups of young people including the homeless, and other out-of-school children and youth; lesbian, gay, bisexual, and transgender (LGBT) youth; orphans, and other children and youth in especially difficult circumstances including those with disability and in juvenile detention, who are often unrepresented or under-represented in population based studies on issues affecting young people [27, 33]. Recently, evidence of school-based studies from subSaharan Africa - and across the African continent, generally - indicates that the population of young people reporting LGBT and other sexual minority orientation is growing [104]. However, studies on their (mental) health needs are limited $[99,100]$.

Recent systematic reviews and primary studies from high-income countries indicate that street-connected children and adolescents represent a good case example of a high-risk group whose self-harm has received inadequate attention in the recent research literature [105]. In carrying out future studies, researchers should clearly define self-harm and more importantly, present to participants the operational definition used in the study, in order to facilitate recall and accurate responses.

More examination is needed of risk-factors for selfharm, not least in attempting to identify the temporal sequence of reported associations to help clarify interdependence between such factors. Future research (including qualitative studies) should also consider exploring factors such as social support, parenting styles, and school climate, which serve to protect young people in sub-Saharan Africa from engaging in self-harm. Such research can inform programmes aimed at strengthening protective and promotive factors within families and schools, and at local community levels can have significant positive effects on improving the developmental outcomes of vulnerable young people [106].
Too much research into risk and protective factors in self-harm has used concepts and measures developed in high income countries. We need to know more about specific features of life in sub-Saharan countries.

\section{Conclusion}

Together, the studies in this review suggest that self-harm is a public (mental) health challenge in young people across countries within sub-Saharan Africa. Given what we know about the link between self-harm and poor mental health, impaired social function and increased suicide risk, more research into the epidemiology, causes and treatment of self-harm in this setting is justified. Too few studies from too few countries have examined the methods of self-harm, risks, protective factors, and the reasons associated with self-harm from a culturally and socially sensitive perspective. The findings of the reviewed studies were overly influenced by the use of pre-existing Western derived models and measures.

\section{Supplementary information}

Supplementary information accompanies this paper at https://doi.org/10. 1186/s12888-020-02587-z.
Additional file 1. PRISMA flow chart.
Additional file 2. PRISMA checklist.
Additional file 3. Search strategies.
Additional file 4. Authors contacted.
Additional file $\mathbf{5}$. Sources of included studies.
Additional file 6. Methods and designs used by studies.
Additional file 7. Methodological quality ratings of studies.

\section{Abbreviations}

IQR: interquartile range; MMAT: mixed method appraisal tool; PRISMA: Preferred Reporting Items for Systematic Reviews and MetaAnalyses; PRISMA-P: Preferred Reporting Items for Systematic Reviews and Meta-Analysis protocols; SA-ETD: South African national Electronic Theses and Dissertations; WHO: World Health Organization; UNICEF: United Nations International Children's Emergency Fund; UNDP: United Nations Development Programme

\section{Acknowledgements}

We wish to thank Judy Wright (a Senior Information Specialist at the University of Leeds) for her assistance with developing comprehensive systematic literature search strategies for this review. We also thank Thirze Hermans, and acknowledge Pauline Oustric in the School of Psychology, University of Leeds, for the translation of eligible French publications into English. We are also grateful to all the suicidologists, authors and self-harm researchers who responded to our correspondence requesting for papers from their personal records for inclusion in this systematic review.

\section{Authors' contributions}

$E Q, M W$ and $\mathrm{AH}$ conceived this study; EQ performed the literature search, extracted the data and conducted narrative analysis; $\mathrm{AH}$ and MW contributed to the eligibility screening consensus discussion to ensure accuracy of data extraction; EQ drafted the manuscript. All the authors contributed to the interpretation of results and revision of the manuscript and approved the final version. 


\section{Funding}

This systematic review was supported by the Leeds International Research Scholarship (LIRS) at the University of Leeds, School of Psychology, in the form of a doctoral scholarship to the first author (EQ) - [Grant №: CFN/sy/ 200631403]. The funder of the study had no role in study design, data collection, data analysis, data interpretation, or writing of the report.

\section{Availability of data and materials}

An unpublished protocol guiding this review was completed in June 2016 by following PRISMA-P statement [107]. A copy of the protocol is available from the first author on reasonable request. All data generated or analysed during this study are included in this published article and its supplementary information files.

\section{Ethics approval and consent to participate}

This systematic review received ethical approval from the School of Psychology Ethics Committee University of Leeds, UK (Ref. №: 16-0373). Consent to participate is not applicable.

\section{Consent for publication}

Not applicable.

\section{Competing interests}

EQ authored two of the cross-sectional studies included in this review. We declare no other competing interests.

\section{Author details}

'Department of Psychology, University of Ghana, P.O. Box LG 84, Legon, Accra, Ghana. ${ }^{2}$ School of Psychology, University of Leeds, Leeds, UK. ${ }^{3}$ Leeds Institute of Health Sciences, University of Leeds, Leeds, UK.

\section{Received: 5 February 2020 Accepted: 5 April 2020}

\section{Published online: 14 May 2020}

\section{References}

1. Platt S, Bille-Brahe U, Kerkhof A, Schmidtke A, Bjerke T, Crepet P, De Leo D, Haring C, Lonnqvist J, Michel K, et al. Parasuicide in Europe: The WHO/EURO multicentre study on parasuicide: I. Introduction and preliminary analysis for 1989. Acta Psychiatrica Scandinavica. 1992;85(2):97-104. https://doi.org/10. 1111/j.1600-0447.1992.tb01451.x..

2. World Health Organization. Practice manual for establishing and maintaining surveillance systems for suicide attempts and self-harm. Geneva: WHO; 2016.

3. Grandclerc S, De Labrouhe D, Spodenkiewicz M, Lachal J, Moro M-R. Relations between nonsuicidal self-injury and suicidal behavior in adolescence: a systematic review. PLoS One. 2016;11(4):e0153760. https:// doi.org/10.1371/journal.pone.0153760.

4. Mars B, Heron J, Klonsky ED, Moran P, O'Connor RC, Tilling K, Wilkinson P, Gunnell D. Predictors of future suicide attempt among adolescents with suicidal thoughts or non-suicidal self-harm: a population-based birth cohort study. Lancet Psychiatry. 2019;6(4):327-37. https://doi.org/10.1016/S22150366(19)30030-6

5. Madge N, Hewitt A, Hawton K, Wilde EJ, Corcoran P, Fekete S, Heeringen $\mathrm{KV}$, Leo DD, Ystgaard M. Deliberate self-harm within an international community sample of young people: comparative findings from the Child \& Adolescent Self-harm in Europe (CASE) study. J Child Psychol Psychiatry. 2008;49(6):667-77. https://doi.org/10.1111/j.1469-7610.2008.01879.x.

6. Muehlenkamp JJ, Claes L, Havertape L, Plener PL. International prevalence of adolescent non-suicidal self-injury and deliberate self-harm. Child Adolesc Psychiatry Ment Health. 2012;6(1):10. https://doi.org/10.1186/1753-2000-6-10.

7. Swannell SV, Martin GE, Page A, Hasking P, St John NJ. Prevalence of nonsuicidal self-injury in nonclinical samples: systematic review, metaanalysis and meta-regression. Suicide Life Threat Behav. 2014;44(3):273-303. https://doi.org/10.1111/sltb.12070.

8. Valencia-Agudo F, Burcher GC, Ezpeleta L, Kramer T. Nonsuicidal self-injury in community adolescents: a systematic review of prospective predictors, mediators and moderators. J Adolesc. 2018;65:25-38. https://doi.org/10. 1016/j.adolescence.2018.02.012.

9. Mortier P, Cuijpers P, Kiekens G, Auerbach R, Demyttenaere K, Green J, Kessler R, Nock M, Bruffaerts R. The prevalence of suicidal thoughts and behaviours among college students: a meta-analysis. Psychol Med. 2018, 48(4):554-65. https://doi.org/10.1017/S0033291717002215.

10. Aggarwal S, Patton G, Reavley N, Sreenivasan SA, Berk M. Youth self-harm in low-and middle-income countries: systematic review of the risk and protective factors. Int J Soc Psychiatry. 2017;63(4):359-75. https://doi.org/10. 1177/0020764017700175.

11. Canetto SS. Suicidal behaviors among Muslim women. Patterns, pathways, meanings, and prevention. Crisis. 2015;36(6):447-58. https://doi.org/10.1027/ 0227-5910/a000347.

12. Mars B, Burrows S, Hjelmeland H, Gunnell D. Suicidal behaviour across the African continent: a review of the literature. BMC Public Health. 2014;14(1): 606. https://doi.org/10.1186/1471-2458-14-606.

13. Liberati A, Altman DG, Tetzlaff J, Mulrow C, Gøtzsche PC, loannidis JP, Clarke M, Devereaux PJ, Kleijnen J, Moher D. The PRISMA statement for reporting systematic reviews and meta-analyses of studies that evaluate health care interventions: explanation and elaboration. PLoS Med. 2009;6(7):e1000100. https://doi.org/10.1371/journal.pmed.1000100.

14. Pienaar E, Grobler L, Busgeeth K, Eisinga A, Siegfried N. Developing a geographic search filter to identify randomised controlled trials in Africa: finding the optimal balance between sensitivity and precision. Health Inform Libraries J. 2011;28(3):210-5. https://doi.org/10.1111/j.1471-1842.2011.00936.x.

15. Davis RHJ. Independent Africa (1960 to present). In: Encyclopedia of African history and culture. Vol. V. New York: Facts on File; 2005.

16. Haddaway NR, Collins AM, Coughlin D, Kirk S. The role of Google scholar in evidence reviews and its applicability to grey literature searching. PLoS One. 2015;10(9):e0138237. https://doi.org/10.1371/journal.pone.0138237.

17. Hong QN, Pluye P, Fàbregues S, Bartlett G, Boardman F, Cargo M, Dagenais P, Gagnon M-P, Griffiths F, Nicolau B. VP26 A critical appraisal tool for systematic mixed studies reviews. Int J Technol Assessment Health Care. 2018;34(S1):166. https://doi.org/10.1017/s0266462318003471.

18. loannidis JP, Patsopoulos NA, Rothstein HR. Reasons or excuses for avoiding meta-analysis in forest plots. BMJ. 2008;336(7658):1413-5. https://doi.org/10. 2307/20510099.

19. Petticrew M, Roberts H. Systematic reviews in the social sciences: a practical guide. Oxford: John Wiley \& Sons; 2006.

20. Omigbodun O, Dogra N, Esan O, Adedokun B. Prevalence and correlates of suicidal behaviour among adolescents in Southwest Nigeria. Int J Soc Psychiatry. 2008;54(1):34-46. https://doi.org/10.1177/0020764007078360.

21. Kapur N, Cooper J, O'Connor RC, Hawton K. Non-suicidal self-injury v. attempted suicide: new diagnosis or false dichotomy? Br J Psychiatry. 2013; 202(5):326-8. https://doi.org/10.1192/bjp.bp.112.116111.

22. Sefa-Dedeh A, Canetto, SS.: Women, Family and suicidal behaviour in Ghana. In: Psychology in international perspective: 50 years of the international council of psychologists. Edited by Gielen UP, Adler, LL., Milgram, NA. Amsterdam/Lisse: Swets \& Zeitlinger B.V.; 1992: 299-309.

23. Wassenaar $D R$, van der Veen MB, Pillay AL. Women in cultural transition: suicidal behavior in south African Indian women. Suicide Life Threat Behav. 1998;28(1):82-93. https://doi.org/10.1111/j.1943-278X.1998.tb00628.x.

24. Beekrum R, Valjee SR, Collings SJ. An emic perspective on the dynamics of non-fatal suicidal behaviour in a sample of south African Indian women. $S$ Afr J Psychol. 2011:41(1):63-73. https://doi.org/10.1177/ 008124631104100107

25. Gage AJ. Association of child marriage with suicidal thoughts and attempts among adolescent girls in Ethiopia. J Adolesc Health. 2013;52(5):654-6. https://doi.org/10.1016/j.jadohealth.2012.12.007.

26. Kritzinger AM. Investigations into adolescent non-fatal suicidal behaviour at a Gauteng public hospital: patient and staff experiences. Doctoral thesis. Pretoria: University of Pretoria; 2018.

27. Meissner BL, Bantjes J. Disconnection, reconnection and autonomy: four young south African men's experience of attempting suicide. J Youth Stud. 2017;20(7):781-97. https://doi.org/10.1080/13676261.2016.1273512.

28. Kinyanda E, Kizza R, Levin J, Ndyanabangi S, Abbo C. Adolescent suicidality as seen in rural northeastern Uganda. Crisis. 2011;32(1):43-51. https://doi. org/10.1027/0227-5910/a000059.

29. Pretorius S. Deliberate self-harm among adolescents in south African children's homes Master Thesis. South Africa: University of Pretoria; 2011.

30. Swahn MH, Palmier JB, Kasirye R, Yao H. Correlates of suicide ideation and attempt among youth living in the slums of Kampala. Int J Environ Res Public Health. 2012;9(2):596-609. https://doi.org/10.3390/ijerph9020596.

31. Asante KO, Meyer-Weitz A. Prevalence and predictors of suicidal ideations and attempts among homeless children and adolescents in Ghana. J Child 
Adolesc Ment Health. 2017;29(1):27-37. https://doi.org/10.2989/17280583. 2017.1287708

32. Shilubane HN, Ruiter RA, Bos AE, van den Borne B, James S, Reddy PS. Psychosocial determinants of suicide attempts among black south African adolescents: a qualitative analysis. J Youth Stud. 2012;15(2):177-89. https:// doi.org/10.1080/13676261.2011.634400.

33. Cheng Y, Li X, Lou C, Sonenstein FL, Kalamar A, Jejeebhoy S, DelanyMoretlwe $\mathrm{S}$, Brahmbhatt $\mathrm{H}$, Olumide AO, Ojengbede $\mathrm{O}$. The association between social support and mental health among vulnerable adolescents in five cities: findings from the study of the well-being of adolescents in vulnerable environments. J Adolesc Health. 2014;55(6):S31-8. https://doi.org/ 10.1016/j.jadohealth.2014.08.020.

34. Flisher A, Ziervogel C, Chalton D, Leger P, Robertson B. Risk-taking behaviour of cape peninsula high-school students. Part II. Suicidal behaviour. S Afr Med J. 1993;83(7):474-6.

35. Kebede D, Ketsela T. Suicide attempts in Ethiopian adolescents in Addis Abeba high schools. Ethiop Med J. 1993;31(2):83-90.

36. Peltzer K, Cherian V, Cherian L. Cross-cultural attitudes towards suicide among south African secondary school pupils. East Afr Med J. 2000;77(3):165-7.

37. Madu S, Matla M. The prevalence of suicidal behaviours among secondary school adolescents in the Limpopo Province, South Africa. S Afr J Psychol. 2003;33(2):126-32. https:/doi.org/10.1177/008124630303300208.

38. Wild LG, Flisher AJ, Lombard C. Suicidal ideation and attempts in adolescents: associations with depression and six domains of self-esteem. J Adolesc. 2004; 27(6):611-24. https:/doi.org/10.1016/j.adolescence.2004.03.001.

39. Sommer M. Suicidal behaviour of high school students: attempts, ideation and risk factors of south African and German adolescents Master' Thesis. South Africa: University of South Africa; 2005.

40. Flisher AJ, Ward CL, Liang H, Onya H, Mlisa N, Terblanche S, Bhana A, Parry CD, Lombard CJ. Injury-related behaviour among south African high-school students at six sites. S Afr Med J. 2006;96(9):825-30.

41. Shiferaw S, Fantahun M, Bekele A. Psychosocial problems among students in preparatory school, in Dessie town, north East Ethiopia. Ethiop J Health Dev. 2006;20(1):47-55

42. Peltzer K. Social support and suicide risk among secondary school students in Cape Town, South Africa. Psychol Rep. 2008;103(3):653-60. https://doi. org/10.2466/pro.103.3.653-660.

43. Mashego TB, Madu S. Suicide-related behaviours among secondary school adolescents in the Welkom and Bethlehem areas of the Free State province (South Africa). S Afr J Psychol. 2009:39(4):489-97. https://doi.org/10.1177/ 008124630903900410.

44. Nanewortor FS. Impact of religiosity, Africentric values and psychological distress on adolescent suicidal ideation. Master Thesis. Ghana: University of Ghana; 2011.

45. Campbell CS. Life stressors and resources as predictors of adolescent suicide attempt Master Thesis. South Africa: University of the Free State; 2012.

46. Van Niekerk L, Scribante L, Raubenheimer P. Suicidal ideation and attempt among south African medical students. South Afr Med J. 2012;102(6):372-3. https://doi.org/10.7196/samj.5503.

47. Vawda NB. Associations between family suicide and personal suicidal behaviour among youth in KwaZulu-Natal, South Africa. South Afr Fam Practc. 2012;54(3):244-9. https://doi.org/10.1080/20786204.2012.10874222.

48. Muula AS, Siziya S, Rudatsikira E. Self-inflicted serious injuries among adolescents in Zambia. Tanzania J Health Res. 2013;15(1):1-8. https://doi. org/10.4314/thrb.v15i1.7.

49. Shilubane HN, Ruiter RA, van den Borne B, Sewpaul R, James S, Reddy PS. Suicide and related health risk behaviours among school learners in South Africa: results from the 2002 and 2008 national youth risk behaviour surveys. BMC Public Health. 2013;13(1):926. https://doi.org/10.1186/1471-2458-13-926.

50. van Rooyen C. An exploratory study of deliberate self-harm in a south African student population Master Thesis. South Africa: University of Pretoria; 2013.

51. Chinawa J, Manyike P, Obu H, Odetunde O, Aniwada E, Ndu I, Chinawa A. Behavioral disorder amongst adolescents attending secondary school in Southeast Nigeria. Behav Neurol. 2014;2014:1-10. https://doi.org/10.1155/ 2014/705835.

52. Lippi C. An exploratory study of the relationship between deliberate selfharm and symptoms of depression and anxiety among a south African university population. Master Thesis. South Africa: University of Pretoria; 2014.
53. Penning SL, Collings SJ. Perpetration, revictimization, and self-injury: traumatic reenactments of child sexual abuse in a nonclinical sample of south African adolescents. J Child Sex Abuse. 2014;23(6):708-26. https://doi. org/10.1080/10538712.2014.931319.

54. Randall JR, Doku D, Wilson ML, Peltzer K. Suicidal behaviour and related risk factors among school-aged youth in the Republic of Benin. PLoS One. 2014; 9(2):e88233. https://doi.org/10.1371/journal.pone.0088233.

55. Shilubane HN, Ruiter RA, Bos AE, van den Borne B, James S, Reddy PS. Psychosocial correlates of suicidal ideation in rural south African adolescents. Child Psychiatry Hum Dev. 2014;45(2):153-62. https://doi.org/ 10.1007/s10578-013-0387-5.

56. Cluver L, Orkin M, Boyes ME, Sherr L. Child and adolescent suicide attempts, suicidal behavior, and adverse childhood experiences in South Africa: a prospective study. J Adolesc Health. 2015;57(1):52-9. https://doi.org/10. 1016/j.jadohealth.2015.03.001.

57. Ng LC, Kirk CM, Kanyanganzi F, Fawzi MCS, Sezibera V, Shema E, Bizimana Jl, Cyamatare FR, Betancourt TS. Risk and protective factors for suicidal ideation and behaviour in Rwandan children. Br J Psychiatry. 2015;207(3):262-8. https://doi.org/10.1192/bjp.bp.114.154591.

58. Giru BW. Prevalence and associated factors of suicidal ideation and attempt among high school adolescent students in Fitche Town, north Shoa, Oromia region, Ethiopia, 2012: Institutional based cross sectional study. J Health Medi Nurs. 2016;23(2016):1-12.

59. Shaikh MA, Lloyd J, Acquah E, Celedonia KL, Wilson ML. Suicide attempts and behavioral correlates among a nationally representative sample of school-attending adolescents in the republic of Malawi. BMC Public Health. 2016;16(1):843. https://doi.org/10.1186/s12889-016-3509-8.

60. van der Walt F. Self-harming behaviour among university students: a south African case study. J Psychol Afr. 2016;26(6):508-12. https://doi.org/10.1080/ 14330237.2016.1250420.

61. Akanni OO, Koleoso ON, Olashore AA, Adayonfo EO, Osundina AF, Ayilara $\mathrm{OO}$. Gender and other risk factors associated with risky behaviours among Nigerian adolescents. J Adolesc. 2017;57:13-7. https://doi.org/10.1016/j. adolescence.2017.03.002

62. Asante KO, Kugbey N, Osafo J, Quarshie EN-B, Sarfo JO. The prevalence and correlates of suicidal behaviours (ideation, plan and attempt) among adolescents in senior high schools in Ghana. SSM-Population Health. 2017;3: 427-34. https://doi.org/10.1016/j.ssmph.2017.05.005.

63. James S, Reddy SP, Ellahebokus A, Sewpaul R, Naidoo P. The association between adolescent risk behaviours and feelings of sadness or hopelessness: a cross-sectional survey of south African secondary school learners. Psychol Health Med. 2017;22(7):778-89. https://doi.org/10.1080/ 13548506.2017.1300669.

64. Nyandindi US. Tanzania Mainland Global School-based Student Health Survey Country Report; 2017.

65. Peltzer K, Pengpid S. Lifestyle and mental health among school-going adolescents in Namibia. J Psychol Afr. 2017;27(1):69-73. https://doi.org/10. 1080/14330237.2016.1268293.

66. Stansfeld SA, Rothon C, Das-Munshi J, Mathews C, Adams A, Clark C, Lund C. Exposure to violence and mental health of adolescents: south African health and well-being study. BJPsych Open. 2017;3(5):257-64. https://doi. org/10.1192/bjpo.bp.117.004861.

67. Amare T, Meseret Woldeyhannes S, Haile K, Yeneabat T. Prevalence and associated factors of suicide ideation and attempt among adolescent high school students in Dangila town, Northwest Ethiopia. Psychiatry J. 2018; 2018. https://doi.org/10.1155/2018/7631453.

68. Khuzwayo N, Taylor M, Connolly C. High risk of suicide among high-school learners in uMgungundlovu district, KwaZulu-Natal Province, South Africa. South Afr Med J. 2018;108(6):517-23. https://doi.org/10.7196/samj.2018. v108i6.12843.

69. Liu X, Huang Y, Liu Y. Prevalence, distribution, and associated factors of suicide attempts in young adolescents: school-based data from 40 lowincome and middle-income countries. PLoS One. 2018;13(12):e0207823. https://doi.org/10.1371/journal.pone.0207823.

70. van der Wal W, George AA. Social support-oriented coping and resilience for self-harm protection among adolescents. J Psychol Afr. 2018;28(3):23741. https://doi.org/10.1080/14330237.2018.1475508.

71. Baiden P, Kuuire VZ, Shrestha N, Tonui BC, Dako-Gyeke M, Peters KK. Bullying victimization as a predictor of suicidal ideation and suicide attempt among senior high school students in Ghana: results from the 2012 Ghana 
global school-based health survey. J Sch Violence. 2019;18(2):300-17. https://doi.org/10.1080/15388220.2018.1486200.

72. Brittain K, Myer L, Phillips N, Cluver LD, Zar HJ, Stein DJ, Hoare J. Behavioural health risks during early adolescence among perinatally HIV-infected south African adolescents and same-age, HIV-uninfected peers. Aids Care. 2019; 31(1):131-40. https://doi.org/10.1080/09540121.2018.1533233.

73. Darré T, Consuela KAC, Saka B, Djiwa T, Ekouévi KD, Napo-Koura G. Suicidal ideation and suicide attempts in subjects aged 15-19 in Lomé (Togo). BMC Res Notes. 2019;12(1):187. https://doi.org/10.1186/s13104-019-4233-0.

74. Koyanagi A, Oh H, Carvalho AF, Smith L, Haro JM, Vancampfort D, Stubbs B, Devylder JE. Bullying victimization and suicide attempt among adolescents aged 12-15 years from 48 countries. J Am Acad Child Adolesc Psychiatry. 2019;58(9):907-18. https://doi.org/10.1016/j.jaac.2018.10.018.

75. Koyanagi A, Stubbs B, Oh H, Veronese N, Smith L, Haro JM, Vancampfort D. Food insecurity (hunger) and suicide attempts among 179,771 adolescents attending school from 9 high-income, 31 middle-income, and 4 lowincome countries: a cross-sectional study. J Affect Disord. 2019;248:91-8. https://doi.org/10.1016/j.jad.2019.01.033.

76. Nguyen KH, Padilla M, Villaveces A, Patel P, Atuchukwu V, Onotu D, Apondi R, Aluzimbi G, Chipimo P, Kancheya N. Coerced and forced sexual initiation and its association with negative health outcomes among youth: results from the Nigeria, Uganda, and Zambia violence against children surveys. Child Abuse Negl. 2019;96:104074. https://doi.org/10.1016/j.chiabu.2019.104074.

77. Quarshie ENB, Cheataa-Plange HV, Annor F, Asare-Doku W, Lartey JKS. Prevalence of suicidal behaviour among nursing and midwifery college students in Ghana. Nursing Open. 2019;6:897-906. https://doi.org/10.1002/ nop2.271.

78. Shayo FK, Lawala PS. Does food insecurity link to suicidal behaviors among in-school adolescents? Findings from the low-income country of subSaharan Africa. BMC Psychiatry. 2019;19(1):227. https://doi.org/10.1186/ s12888-019-2212-6.

79. Thornton VJ, Asanbe CB, Denton ED. Clinical risk factors among youth at high risk for suicide in South Africa and Guyana. Depression Anxiety. 2019; 36(5):423-32. https://doi.org/10.1002/da.22889.

80. Tolulope O, Olutayo A, Babatunde S, Adesanmi A. Suicidality in a nonclinical sample of Nigerian adolescents: prevalence and correlates. Suicidology Online. 2019;10(7):1-8.

81. Uddin R, Burton NW, Maple M, Khan SR, Khan A. Suicidal ideation, suicide planning, and suicide attempts among adolescents in 59 low-income and middle-income countries: a population-based study. Lancet Child Adolescent Health. 2019;3(4):223-33. https://doi.org/10.1016/s23524642(18)30403-6.

82. Vancampfort D, Stubbs B, Mugisha J, Firth J, Van Damme T, Smith L, Koyanagi A. Leisure-time sedentary behavior and suicide attempt among 126,392 adolescents in 43 countries. J Affect Disord. 2019;250:346-53. https://doi.org/10.1016/j.jad.2019.03.053.

83. Cummins R, Allwood C. Suicide attempts or threats by children and adolescents in Johannesburg. S Afr Med J. 1984;66:726-9.

84. Schlebusch L. Self-destructive behaviour in adolescents. S Afr Med J. 1985; 68(11):792-5.

85. Pillay AL. Factors precipitating parasuicide among young south African Indians. Psychol Rep. 1987;61 (2):545-6. https://doi.org/10.2466/pro.1987.61.2.545.

86. Pillay AL. Methods of self-destructive behaviour in adolescents and young adults. Psychol Rep. 1988;63(2):552-4. https://doi.org/10.2466/pr0.1988.63.2.552.

87. Pillay AL, Wassenaar DR. Rescue expectations and hopelessness in adolescent parasuicides. Percept Mot Skills. 1991;72(2):363-6. https:/doi.org/ 10.2466/pms.1991.72.2.363.

88. Mhlongo T, Peltzer K. Parasuicide among youth in a general hospital in South Africa. Curationis. 1999;22(2):72-6. https://doi.org/10.4102/curationis.V22i2.735.

89. Yéo-Tenena YJ, Yao YP, Bakayoko AS, N'dja GR, Kouamé LM, Soro SJ, Delafosse RC. Étude descriptive des conduites suicidaires des adolescents à Abidjan (Descriptive study of suicidal adolescents conducted in Abidjan). L'Encephale. 2010;36:D41-7. https://doi.org/10.1016/j.encep.2008.08.007.

90. Okoko AR, Ekouya Bowassa G, Oko APG, Mbika-Cardorelle A, Dzalamou M, Moyen GM. Tentatives de suicide de l'enfant et de l'adolescent a’ Brazzaville (Suicide attempts by children and teenagers in Brazzaville). Archives de Pediatrie. 2011;18(7):810-1. https://doi.org/10.1016/j.arcped.2011.04.018.

91. Fine $G$, Alison $H$, Van der Westhuizen $D$, Krüger $C$. Predicting frequency of suicide attempts of adolescent outpatients at Weskoppies hospital using clinical and demographic characteristics. S Afr J Psychiatry. 2012;18(1):22-6. https://doi.org/10.4102/sajpsychiatry.v18i1.271.
92. Devries KM, Mak JY, Bacchus LJ, Child JC, Falder G, Petzold M, Astbury J, Watts $\mathrm{CH}$. Intimate partner violence and incident depressive symptoms and suicide attempts: a systematic review of longitudinal studies. PLoS Med. 2013;10(5):e1001439. https://doi.org/10.1371/journal.pmed.1001439.

93. Serafini G, Canepa G, Adavastro G, Nebbia J, Murri MB, Erbuto D, Pocai B, Fiorillo A, Pompili M, Flouri E, et al. The relationship between childhood maltreatment and non-suicidal self-injury: a systematic review. Front Psychiatry. 2017;8. https://doi.org/10.3389/fpsyt.2017.00149.

94. Liu RT, Scopelliti KM, Pittman SK, Zamora AS. Childhood maltreatment and non-suicidal self-injury: a systematic review and meta-analysis. Lancet Psychiatry. 2018;5(1):51-64. https://doi.org/10.1016/s2215-0366(17)30469-8.

95. Pillay AL, Wassenaar DR. Family dynamics, hopelessness and psychiatric disturbance in parasuicidal adolescents. Aust N Z J Psychiatry. 1997;31(2): 227-31. https://doi.org/10.3109/00048679709073825.

96. Madu SN, Matla M-QP. Family environmental factors as correlates for adolescent suicidal behaviors in the Limpopo Province of South Africa. Soc Behav Pers Int J. 2004;32(4):341-53. https://doi.org/10.2224/sbp.2004.32.4.341

97. Carvalho AF, Stubbs B, Vancampfort D, Kloiber S, Maes M, Firth J, Kurdyak PA, Stein DJ, Rehm J, Koyanagi A. Cannabis use and suicide attempts among 86,254 adolescents aged 12-15 years from 21 low-and middleincome countries. European Psychiatry. 2019;56:8-13. https://doi.org/10. 1016/j.eurpsy.2018.10.006.

98. Osafo J, Akotia CS, Andoh-Arthur J, Quarshie EN-B. Attempted suicide in Ghana: motivation, stigma, and coping. Death Studies. 2015;39(5):274-80. https://doi.org/10.1080/07481187.2014.991955.

99. Kabiru CW, Izugbara CO, Beguy D. The health and wellbeing of young people in sub-Saharan Africa: an under-researched area? BMC Int Health Hum Rights. 2013;13(1):11. https://doi.org/10.1186/1472-698x-13-11.

100. Patton GC, Sawyer SM, Santelli JS, Ross DA, Afifi R, Allen NB, Arora M, Azzopardi P, Baldwin W, Bonell C. Our future: a lancet commission on adolescent health and wellbeing. Lancet. 2016;387(10036):2423-78. https:// doi.org/10.1016/S0140-6736(16)00579-1.

101. Petroni S, Patel V, Patton G. Why is suicide the leading killer of older adolescent girls? Lancet. 2015;386(10008):2031-2. https://doi.org/10.1016/ S0140-6736(15)01019-3.

102. Kizza D, Knizek B, Kinyanda E, Hjelmeland H. An escape from agony: a qualitative psychological autopsy study of women's suicide in a postconflict northern Uganda. Int J Qual Stud Health Well Being. 2012;7(1): 18463. https://doi.org/10.3402/ghw.v7i0.18463.

103. Adinkrah M. Better dead than dishonored: masculinity and male suicidal behavior in contemporary Ghana. Soc Sci Med. 2012;74(4):474-81. https:// doi.org/10.1016/j.socscimed.2010.10.011.

104. Mucherah W, Owino E, McCoy K. Grappling with the issue of homosexuality: perceptions, attitudes, and beliefs among high school students in Kenya. Psychol Res Behav Manag. 2016;9:253-62. https://doi.org/10.2147/PRBM. S112421.

105. Woan J, Lin J, Auerswald C. The health status of street children and youth in low-and middle-income countries: a systematic review of the literature. J Adolescent Health. 2013;53(3):314-21. e312. https://doi.org/10.1016/j. jadohealth.2013.03.013.

106. Patel V, Flisher AJ, Nikapota A, Malhotra S. Promoting child and adolescent mental health in low and middle income countries. J Child Psychol Psychiatry. 2008;49(3):313-34. https://doi.org/10.1111/j.1469-7610.2007. 01824.x.

107. Moher D, Shamseer L, Clarke M, Ghersi D, Liberati A, Petticrew M, Shekelle P, Stewart LA. Preferred reporting items for systematic review and metaanalysis protocols (PRISMA-P) 2015 statement. Syst Rev. 2015;4(1):1. https:// doi.org/10.1186/2046-4053-4-1.

\section{Publisher's Note}

Springer Nature remains neutral with regard to jurisdictional claims in published maps and institutional affiliations. 